\title{
An Eulerian finite-volume scheme for large elastoplastic deformations in solids
}

\author{
P. T. Barton, D. Drikakis* \& E. I. Romenski ${ }^{\dagger}$ \\ Department of Aerospace Sciences, Cranfield University, Cranfield, Bedfordshire, MK43 OAL, UK
}

\begin{abstract}
SUMMARY
Conservative formulations of the governing laws of elastoplastic solid media have distinct advantages when solved using high-order shock capturing methods for simulating processes involving large deformations and shock waves. In this paper one such model is considered where inelastic deformations are accounted for via conservation laws for elastic strain with relaxation source terms. Plastic deformations are governed by the relaxation time of tangential stresses. Compared to alternative Eulerian conservative models the governing system consists of fewer equations overall. A numerical scheme for the inhomogeneous system is proposed based upon temporal splitting. In this way the reduced system of non-linear elasticity is solved explicitly, with convective fluxes evaluated using highorder approximations of Riemann problems locally throughout the computational mesh. Numerical stiffness of the relaxation terms at high strain-rates is avoided by utilising certain properties of the governing model and performing an implicit update. The methods are demonstrated using testcases involving large deformations and high strain-rates in one-, two-, and three-dimensions.
\end{abstract}

KEY WORDS: Elastic-plastic solids; finite-volume; high-resolution schemes, Riemann solver; WENO

\section{INTRODUCTION}

Under high strain-rate dynamic loading solid materials exhibit complex non-linear behaviour such as split elastic-plastic shock waves transporting large deformations. From the viewpoint of numerical modelling this represents a challenge since governing models must, in the very least, account for the materials ability to resist shear loads, and accurate models consist of highly non-linear constitutive equations.

Various models and numerical methods have been developed to simulate processes in solid media. The choice of these methods will depend largely on the problem and the available resources. Benson [3] provides a detailed review of the most common of these. Lagrangian methods, where the computational grid conforms to the material, are efficient and can

\footnotetext{
*Correspondence to: d.drikakis@cranfield.ac.uk

${ }^{\dagger}$ On leave from Sobolev Institute of Mathematics, Russian Academy of Sciences, Novosibirsk, Russia

Contract/grant sponsor: Publishing Arts Research Council; contract/grant number: 98-1846389
} 
resolve material interfaces exactly. However, in circumstances where the material undergoes large distortions, accuracy cannot be guaranteed and in some cases the methods can fail. Eulerian methods on the other hand use fixed grids and allow the material to flow through it. Traditionally, Eulerian methods are based on the finite element or finite difference methods, requiring the explicit inclusion of artificial viscosity to avoid numerical artifacts in problems involving shock waves. Whilst this works in practice it is desirable to introduce the necessary viscosity in a more natural way.

In view of the complex behaviour that can occur in solid materials, the requirements from the employed numerical algorithms is akin to those of hydrodynamics: the algorithm must be capable of handling large distortions and achieve high wave resolution. High-order shock capturing methods, based upon solving Riemann problems locally at each cell edge throughout a computational domain, have emerged as a favourable approach to meeting these requirements. Their success in fluid mechanics has led to a wealth of research and consequently methods of increasing accuracy and efficiency are now available. Many of these numerical tools are derived on the basis of arbitrary systems of conservation laws, thus retrofitting these techniques to other physics disciplines is possible given that the governing models are written in adequate form.

Application of these numerical tools to solid mechanics has been made possible by formulations of the governing theory as first-order hyperbolic systems of conservation laws in the Eulerian frame of reference $[6,12,21,8]$. Neglecting inelastic deformations, the special case of non-linear elasticity is governed by a homogeneous system of partial-differential-equations, conserving mass, momentum, strain and energy, in conjunction with compatibility constraints. Developments of Godunov methods for such formulations include the works of LeFloch and Olsson [13] and Titarev et al [25]. One immediate advantage of such schemes is the inclusion of material strength in the Riemann problem solution.

Inelastic deformations can be accounted for by modifying the governing model in a number of ways. The multiplicative decomposition of the total deformation tensor into elastic and plastic parts can be used to yield additional conservation laws for the plastic deformation tensor [12, 21]. Developments using these models include the work of Vorobiev et al [26], Wang et al [28], Walter et al [27], and Miller and Colella [17]. A disadvantage of this approach is the additional expense of solving a much larger system of equations. In a different approach, a modified elastic potential was proposed in [11] such that the resultant constitutive equations for stress obey von Mises yield criterion, but is restricted to idealised plasticity theory. In the present study the elastic-plastic formulation of Godunov and Romenski [8] is used, where plasticity is introduced via source terms for the equations of the elastic deformation tensor.

In another study by the present authors [2] a high-order Godunov method was developed for the model of Godunov and Romenski [8], using the special case of non-linear elasticity in one space dimension. Numerical results were compared to exact solutions of initial value problems involving three-dimensional deformations. High-order accuracy was achieved using monotonicity-preserving weighted essentially non-oscillatory (MPWENO) reconstruction. Extension of these methods to inelastic deformations and multi-dimensions using this model requires little modifications of the method for discretizing convective fluxes. The main difficulty becomes the evaluation of source terms of the now inhomogeneous system.

The rest of this paper is organised as follows. In Section 2 the governing theories are reviewed, whilst Section 3 details the eigenstructure for the three dimensional system. Section 4 discusses the necessary constitutive models. In Section 5 the numerical scheme is proposed, and in 
Section 6 these are tested using example testcases in one-, two- and three-dimensions. Finally in Section 7 a concluding summary is given.

\section{GOVERNING THEORY}

In the model of Godunov and Romenskiy $[6,8]$, the state of a solid is characterised by the elastic deformation gradient $F_{i j}=\partial x_{i} / \partial x_{0_{j}}$ (where $x_{i}$ and $x_{0_{j}}$ denote the spatial and material coordinates of the unstressed reference state respectively), velocity $u_{i}$, and entropy $S$. One has the equations for conservation of momentum and energy

$$
\begin{gathered}
\frac{\partial \rho u_{i}}{\partial t}+\frac{\partial\left(\rho u_{i} u_{k}-\sigma_{i k}\right)}{\partial x_{k}}=0, \\
\frac{\partial \rho E}{\partial t}+\frac{\partial\left(\rho u_{k} E-u_{i} \sigma_{i k}\right)}{\partial x_{k}}=0,
\end{gathered}
$$

and equations for $F_{i j}$ providing the conservation of strain

$$
\frac{\partial \rho F_{i j}}{\partial t}+\frac{\partial\left(\rho F_{i j} u_{k}-\rho F_{k j} u_{i}\right)}{\partial x_{k}}=-u_{i} \beta_{j}-\varphi_{i j}
$$

Here $E=\left(\mathscr{E}+|u|^{2} / 2\right)$ is the total energy, with $\mathscr{E}$ the internal energy. Density and the stress tensor are given by

$$
\begin{aligned}
\rho & =\rho_{0} / \operatorname{det}|F|, \\
\sigma_{i j} & =\rho F_{i k} \frac{\partial \mathscr{E}}{\partial F_{j k}},
\end{aligned}
$$

where $\rho_{0}$ denotes the density of the initial unstressed medium. The terms on the right-handside of Eq. (3) are given by

$$
\begin{gathered}
\beta_{j}=\frac{\partial \rho F_{k j}}{\partial x_{k}}, \\
\varphi_{i j}=\frac{1}{2 G \tau} \sigma_{i k}^{\prime} \rho F_{k j},
\end{gathered}
$$

where $\sigma_{i k}^{\prime}=\sigma_{i k}-\sigma_{m m} \delta_{i k} / 3$ is the tensor of deviatoric stress, $\delta_{i k}$ is the Kronecker delta, and $G(\rho, S)$ is the shear modulus which can depend on the state of the material. The vector $\beta$ is an artificial vector variable providing the conservative form of equations for $F$. The tensor $\varphi$ provides the necessary mechanisims for decaying deviatoric stress via Maxwell's relaxation model; the relaxation time, $\tau$, governs the rate of decay of deviatoric stress. For the case of small deformations a corresponding rheological law reads as

$$
\frac{\mathrm{d} \sigma_{i j}^{\prime}}{\mathrm{d} t}=2 G \dot{\varepsilon}_{i j}^{\prime}-\frac{\sigma_{i j}^{\prime}}{\tau}
$$

where $\dot{\varepsilon}_{i j}^{\prime}$ is the strain rate deviator.

The vector $\beta$ satisfies an additional conservation-form equation

$$
\frac{\partial \beta_{j}}{\partial t}+\frac{\partial\left(u_{k} \beta_{j}+\varphi_{k j}\right)}{\partial x_{k}}=0 .
$$


Owing to this equation $u_{i} \beta$ in Eq. (3) can be treated as source terms in the governing equations.

Note that in the case of pure elastic processes there is no plastic deformations and it is necessary to set $\varphi=0$. Moreover if $\varphi=0$, then $\beta=0$ if it is equal to 0 in the initial data. It is necessary only to keep in mind that the solutions to the reduced elasticity system must satisfy the compatibility constraints

$$
\frac{\partial \rho F_{k j}}{\partial x_{k}}=0
$$

It is noted that the conservation laws of non-linear elasticity expressed in terms of the Lagrangian elastic deformation tensor $f=F^{-1}$ satisfy the six compatibility conditions

$$
\frac{\partial f_{m n}}{\partial x_{l}}-\frac{\partial f_{m l}}{\partial x_{n}}=0
$$

Eq. (10) follows from (11) since the following relationship can be found

$$
\frac{\partial \rho F_{k j}}{\partial x_{k}}=\rho F_{i j} F_{n m}\left(\frac{\partial f_{m n}}{\partial x_{i}}-\frac{\partial f_{m i}}{\partial x_{n}}\right),
$$

For the case of inelastic deformations, the conservation laws expressed in terms of $f[21,17]$ satisfy six additional unsteady laws (see [8]). Using Eq. (12) along with the conservation laws for $F$ above it is possible to show that Eq. (9) is a consequence of these.

The system is closed by analytic formulae for the specific internal energy and relaxation time in terms of the parameters of state

$$
\begin{gathered}
\mathscr{E}=\mathscr{E}\left(F_{i j}, S\right), \\
\tau=\tau\left(\sigma_{i j}, T\right),
\end{gathered}
$$

where $T=\partial \mathscr{E} / \partial S$ is temperature.

The complete three dimensional system forms a hyperbolic system of conservation laws in Cartesian coordinates. In matrix form

$$
\frac{\partial U}{\partial t}+\frac{\partial \mathscr{F}^{\alpha}}{\partial x_{\alpha}}=-S^{c}-S^{p}
$$

with

$$
\begin{array}{r}
U=\left(\begin{array}{c}
\rho u \\
\rho F^{T} e_{1} \\
\rho F^{T} e_{2} \\
\rho F^{T} e_{3} \\
\rho E
\end{array}\right), \quad \mathscr{F}^{\alpha}=\left(\begin{array}{c}
u_{\alpha} \rho u-\sigma e_{\alpha} \\
u_{\alpha} \rho F^{T} e_{1}-u_{1} \rho F^{T} e_{\alpha} \\
u_{\alpha} \rho F^{T} e_{2}-u_{2} \rho F^{T} e_{\alpha} \\
u_{\alpha} \rho F^{T} e_{3}-u_{3} \rho F^{T} e_{\alpha} \\
u_{\alpha} \rho E-(\sigma u) e_{\alpha}
\end{array}\right) . \\
S^{c}=\left(\begin{array}{c}
u_{1}(\nabla \cdot \rho F) \\
u_{2}(\nabla \cdot \rho F) \\
u_{3}(\nabla \cdot \rho F) \\
0
\end{array}\right), \quad S^{p}=\frac{1}{2 G \tau}\left(\begin{array}{c}
\left(\sigma^{\prime} \cdot \rho F\right) e_{1} \\
\left(\sigma^{\prime} \cdot \rho F\right) e_{2} \\
\left(\sigma^{\prime} \cdot \rho F\right) e_{3} \\
0
\end{array}\right),
\end{array}
$$

where $e_{\alpha}$ are the Cartesian unit vectors

This particular formulation is a result of the work in [23] where it is shown how to properly include the source terms associated with inelasticity to yield a thermodynamically compatible 
system of equations, i.e. its solutions satisfy thermodynamic laws. Using Eqs. (3)-(4),(6)-(7) it is possible to show that the combination of equations governing the conservation of strain conserve mass, by means of recovering the continuity equation

$$
\frac{\partial \rho}{\partial t}+\frac{\partial \rho u_{k}}{\partial x_{k}}=0
$$

(see appendix for the proof). Further this equation is used in the development of numerical methods instead of one equation for deformation gradient in order to provide conservation of mass. As a consequence of (15) the entropy balance law can be derived in the form

$$
\frac{\partial \rho S}{\partial t}+\frac{\partial \rho S u_{k}}{\partial x_{k}}=Q
$$

Here $Q$ is the entropy production, which is nonnegative due to the choice of the inelastic deformation source terms (17):

$$
Q=\frac{1}{2 G \tau T} \sigma_{i j}^{\prime} \sigma_{j i}^{\prime} \geq 0
$$

\section{CHARACTERISTIC DECOMPOSITION}

In the ensuing computational method the convective flux terms in Eq. (15) are discretized using the well known method of Godunov. The solution is therefore required of a local Riemann problem at the boundaries of each cell in the computational mesh. The solution is found using an approximate method based upon characterstic tracing and thus requires detailed knowledge of the eigenvalues and eigenvectors of (15).

Introducing the vector of primitive variables $W=\left(u, F^{T} e_{1}, F^{T} e_{2}, F^{T} e_{3}, S\right)$, Eq. (15) can be rewritten as a quasi-linear system

$$
\frac{\partial W}{\partial t}+\mathscr{A}^{\alpha} \frac{\partial W}{\partial x_{\alpha}}=-S^{p}
$$

The Jacobian appearing in Eq. (21) is

$$
\mathscr{A}^{\alpha}=\left(\begin{array}{ccccc}
u_{\alpha} I & -A^{\alpha 1} & -A^{\alpha 2} & -A^{\alpha 3} & -B^{\alpha} \\
-F^{T} D_{\alpha 1} & u_{\alpha} I & 0 & 0 & 0 \\
-F^{T} D_{\alpha 2} & 0 & u_{\alpha} I & 0 & 0 \\
-F^{T} D_{\alpha 3} & 0 & 0 & u_{\alpha} I & 0 \\
0 & 0 & 0 & 0 & u_{\alpha}
\end{array}\right),
$$

where $D_{i j}=e_{i} \otimes e_{j}^{T}$ represents the unit dyads and the coefficients are given by

$$
A_{i j}^{\alpha \beta}=\frac{1}{\rho} \frac{\partial \sigma_{\alpha i}}{\partial F_{\beta j}}, \quad B_{i}^{\alpha}=\frac{1}{\rho} \frac{\partial \sigma_{\alpha i}}{\partial S} .
$$

The vector of source terms is

$$
S^{p}=\frac{1}{2 G \tau}\left(\begin{array}{c}
0 \\
\left(\sigma^{\prime} F\right) e_{1} \\
\left(\sigma^{\prime} F\right) e_{2} \\
\left(\sigma^{\prime} F\right) e_{3} \\
-\frac{1}{\rho T} \sum_{i, k=1}^{3} \sigma_{i k}^{\prime} \sigma_{i k}
\end{array}\right)
$$




\section{1. $x_{1}$-direction}

For the purpose of derivation the eigenstructure for the flux component aligned with the $x_{1}$ spatial axis is first investigated. For simplicity the suffix $\alpha$ shall be omitted and only the one dimensional counterpart, $\alpha=1$ is considered. If $\lambda$ denotes the wavespeeds then the characteristic polynomial for $(22)(|A-\lambda I|=0)$ has the form

$$
(u-\lambda)^{7} \operatorname{det}\left|\Omega-(u-\lambda)^{2} I\right|=0,
$$

where $\Omega$ is the acoustic tensor

$$
\Omega_{i j}=\left(e_{i}^{T} A_{1 j}\right) \cdot\left(F^{T} e_{1}\right), \quad 1 \leq i, j \leq 3 .
$$

Due to the hyperbolicity of the system (15) $\Omega$ is positive definite and the diagonal matrix of positive eigenvalues can be defined $\mathscr{D}=\operatorname{diag}\left(\sqrt{\lambda_{a c_{1}}}, \sqrt{\lambda_{a c_{2}}}, \sqrt{\lambda_{a c_{3}}}\right)$, with $\lambda_{a c_{3}} \leq \lambda_{a c_{2}} \leq$ $\lambda_{a c_{1}}$, and the orthogonal matrix $Q$, such that (26) can be rewritten

$$
\Omega=Q^{-1} \mathscr{D}^{2} Q .
$$

The diagonal matrix of eigenvalues is thus given by (assuming the order $u_{1}-\sqrt{\lambda_{a c_{1}}} \leq$ $\left.u_{1}-\sqrt{\lambda_{a c_{2}}} \leq \ldots \leq u_{1} \leq \ldots \leq u_{1} I+\sqrt{\lambda_{a c_{1}}}\right)$

$$
\Lambda=\operatorname{diag}\left(u_{1} I-\mathscr{D}, u_{1} I, u_{1} I, u_{1}, u_{1} I+\pi \mathscr{D} \pi\right),
$$

where the permutation matrix

$$
\pi=\left(\begin{array}{lll}
0 & 0 & 1 \\
0 & 1 & 0 \\
1 & 0 & 0
\end{array}\right),
$$

has been used. The matrix of left eigenvectors is

$$
L=\left(l_{1}, l_{2}, l_{3}, l_{4}, l_{5}\right)^{T},
$$

where

$$
\begin{aligned}
& l_{1}=\left(\mathscr{D} Q, Q A_{11}, Q A_{12}, Q A_{13}, Q A_{1}^{0}\right), \\
& l_{2}=\left(0, \frac{1}{F_{11}}\left(F_{12} D_{11}+F_{13} D_{21}\right)-D_{12}-D_{23}, \frac{1}{F_{11}}\left(F_{12} D_{31}\right)-D_{32}, 0,0\right), \\
& l_{3}=\left(0,0, \frac{1}{F_{11}}\left(F_{13} D_{11}\right)-D_{13}, \frac{1}{F_{11}}\left(F_{12} D_{21}+F_{13} D_{31}\right)-D_{22}-D_{33}, 0\right), \\
& l_{4}=\left(0,0,0, \frac{1}{F_{11}}\left(F_{12} D_{21}+F_{13} D_{31}\right)-D_{22}-D_{33}, 0\right), \\
& l_{5}=(0,0,0,0,1) .
\end{aligned}
$$

Using the assumption that the right eigenvectors are orthonormal to the left $(R L=I)$, the matrix of right eigenvectors is

$$
R=\left(r_{1}, r_{2}, r_{3}, r_{4}, r_{5}\right),
$$


where

$$
\begin{aligned}
r_{1}= & \left(\frac{1}{2} Q^{-1} \mathscr{D}^{-1}, \frac{1}{2}\left(F^{T} e_{1}\right) \otimes\left(e_{1}^{T} Q^{-1} \mathscr{D}^{-2}\right),\right. \\
& \left.\frac{1}{2}\left(F^{T} e_{1}\right) \otimes\left(e_{2}^{T} Q^{-1} \mathscr{D}^{-2}\right), \frac{1}{2}\left(F^{T} e_{1}\right) \otimes\left(e_{3}^{T} Q^{-1} \mathscr{D}^{-2}\right), 0\right)^{T}, \\
r_{2}= & \left(0,\left(F^{T} e_{1}\right) \otimes\left(e_{1}^{T} \Psi_{1}\right)-D_{21}-D_{32},\left(F^{T} e_{1}\right) \otimes\left(e_{2}^{T} \Psi_{1}\right)-D_{23},\right. \\
& \left.\left(F^{T} e_{1}\right) \otimes\left(e_{3}^{T} \Psi_{1}\right), 0\right)^{T}, \\
r_{3}= & \left(0,\left(F^{T} e_{1}\right) \otimes\left(e_{1}^{T} \Psi_{2}\right),\left(F^{T} e_{1}\right) \otimes\left(e_{2}^{T} \Psi_{2}\right)-D_{31},\right. \\
& \left.\left(F^{T} e_{1}\right) \otimes\left(e_{3}^{T} \Psi_{2}\right)-D_{22}-D_{33}, 0\right)^{T}, \\
r_{4}= & \left(0,\left(e_{1}^{T} \Omega^{-1} A_{1}^{0}\right) F^{T} e_{1},\left(e_{2}^{T} \Omega^{-1} A_{1}^{0}\right) F^{T} e_{1},\left(e_{3}^{T} \Omega^{-1} A_{1}^{0}\right) F^{T} e_{1}, 1\right)^{T}, \\
r_{5}= & \left(\frac{1}{2} Q^{-1} \mathscr{D}^{-1} \pi,-\frac{1}{2}\left(\left(F^{T} e_{1}\right) \otimes\left(e_{1}^{T} Q^{-1} \mathscr{D}^{-2}\right)\right) \pi,\right. \\
& \left.-\frac{1}{2}\left(\left(F^{T} e_{1}\right) \otimes\left(e_{2}^{T} Q^{-1} \mathscr{D}^{-2}\right)\right) \pi,-\frac{1}{2}\left(\left(F^{T} e_{1}\right) \otimes\left(e_{3}^{T} Q^{-1} \mathscr{D}^{-2}\right)\right) \pi, 0\right)^{T},
\end{aligned}
$$

and

$$
\begin{aligned}
& \Psi_{1}=\Omega^{-1}\left(A_{11} D_{21}+A_{11} D_{32}+A_{12} D_{23}\right), \\
& \Psi_{2}=\Omega^{-1}\left(A_{12} D_{31}+A_{13} D_{22}+A_{13} D_{33}\right) .
\end{aligned}
$$

\section{2. $x_{2}$ - and $x_{3}$-directions}

Whilst it is straightforward to generalise the above eigenvectors to the Jacobians of the fluxes aligned with the other coordinate directions $\left(x_{2}, x_{3}\right)$, it was found in the course of computation that these do not lend themselves to a formulation convenient for numerical computation. This is because the ratios of components of the deformation gradient appearing in the left eigenvectors could, in some special cases lead to a divide by zero. For example, in the case of a uniaxial deformation all non-diagonal elements of the deformation tensor will be equal to zero. Using the above structure on the other coordinate directions results in some of these components appearing as a denominator in the left eigenvectors. It is mentioned that this problem does not arise in the two-dimensional formulation (see [25]). To overcome this problem small modifications can be made to the eigenvectors for each of the coordinate directions.

In the $x_{2}$ direction the left eigenvectors are

$$
\begin{aligned}
& l_{1}=\left(\mathscr{D} Q, Q A^{21}, Q A^{22}, Q A^{23}, Q B^{2}\right), \\
& l_{2}=\left(0, \frac{1}{F_{22}}\left(F_{21} D_{12}+F_{23} D_{22}\right)-D_{11}-D_{23}, \frac{1}{F_{22}}\left(F_{21} D_{32}\right)-D_{31}, 0,0\right), \\
& l_{3}=\left(0,0, \frac{1}{F_{22}}\left(F_{23} D_{12}\right)-D_{13}, \frac{1}{F_{22}}\left(F_{21} D_{22}+F_{23} D_{32}\right)-D_{21}-D_{33}, 0\right), \\
& l_{4}=(0,0,0,0,1), \\
& l_{5}=\left(\pi \mathscr{D} Q,-\pi Q A^{21},-\pi Q A^{22},-\pi Q A^{23},-\pi Q B^{2}\right),
\end{aligned}
$$


and the corresponding right eigenvectors

$$
\begin{aligned}
r_{1}= & \left(\frac{1}{2} Q^{-1} \mathscr{D}^{-1}, \frac{1}{2}\left(F^{T} e_{2}\right) \otimes\left(e_{1}^{T} Q^{-1} \mathscr{D}^{-2}\right)\right. \\
& \left.\frac{1}{2}\left(F^{T} e_{2}\right) \otimes\left(e_{2}^{T} Q^{-1} \mathscr{D}^{-2}\right), \frac{1}{2}\left(F^{T} e_{2}\right) \otimes\left(e_{3}^{T} Q^{-1} \mathscr{D}^{-2}\right), 0\right)^{T}, \\
r_{2}= & \left(0,\left(F^{T} e_{2}\right) \otimes\left(e_{1}^{T} \Psi_{3}\right)-D_{11}-D_{32},\left(F^{T} e_{2}\right) \otimes\left(e_{2}^{T} \Psi_{3}\right)-D_{13},\right. \\
& \left.\left(F^{T} e_{2}\right) \otimes\left(e_{3}^{T} \Psi_{3}\right), 0\right)^{T} \\
r_{3}= & \left(0,\left(F^{T} e_{2}\right) \otimes\left(e_{1}^{T} \Psi_{4}\right),\left(F^{T} e_{2}\right) \otimes\left(e_{2}^{T} \Psi_{4}\right)-D_{31},\right. \\
& \left.\left(F^{T} e_{2}\right) \otimes\left(e_{3}^{T} \Psi_{4}\right)-D_{12}-D_{33}, 0\right)^{T} \\
r_{4}= & \left(0,\left(e_{1}^{T} \Omega^{-1} B^{2}\right) F^{T} e_{2},\left(e_{2}^{T} \Omega^{-1} B^{2}\right) F^{T} e_{2},\left(e_{3}^{T} \Omega^{-1} B^{2}\right) F^{T} e_{2}, 1\right)^{T}, \\
r_{5}= & \left(\frac{1}{2} Q^{-1} \mathscr{D}^{-1} \pi,-\frac{1}{2}\left(\left(F^{T} e_{2}\right) \otimes\left(e_{1}^{T} Q^{-1} \mathscr{D}^{-2}\right)\right) \pi\right. \\
& \left.-\frac{1}{2}\left(\left(F^{T} e_{2}\right) \otimes\left(e_{2}^{T} Q^{-1} \mathscr{D}^{-2}\right)\right) \pi,-\frac{1}{2}\left(\left(F^{T} e_{2}\right) \otimes\left(e_{3}^{T} Q^{-1} \mathscr{D}^{-2}\right)\right) \pi, 0\right)^{T},
\end{aligned}
$$

with

$$
\begin{aligned}
& \Psi_{3}=\Omega^{-1}\left(A^{21} D_{11}+A^{21} D_{32}+A^{22} D_{13}\right), \\
& \Psi_{4}=\Omega^{-1}\left(A^{22} D_{31}+A^{23} D_{12}+A^{23} D_{33}\right) .
\end{aligned}
$$

Similarly for the $x_{3}$ direction the left eigenvectors are

$$
\begin{aligned}
& l_{1}=\left(\mathscr{D} Q, Q A^{31}, Q A^{32}, Q A^{33}, Q B^{3}\right), \\
& l_{2}=\left(0, \frac{1}{F_{33}}\left(F_{31} D_{13}+F_{32} D_{23}\right)-D_{11}-D_{22}, \frac{1}{F_{33}}\left(F_{31} D_{33}\right)-D_{31}, 0,0\right), \\
& l_{3}=\left(0,0, \frac{1}{F_{33}}\left(F_{32} D_{13}\right)-D_{12}, \frac{1}{F_{33}}\left(F_{31} D_{23}+F_{32} D_{33}\right)-D_{21}-D_{32}, 0\right), \\
& l_{4}=(0,0,0,0,1), \\
& l_{5}=\left(\pi \mathscr{D} Q,-\pi Q A^{31},-\pi Q A^{32},-\pi Q A^{33},-\pi Q B^{3}\right),
\end{aligned}
$$

and the corresponding right eigenvectors

$$
\begin{aligned}
r_{1}= & \left(\frac{1}{2} Q^{-1} \mathscr{D}^{-1}, \frac{1}{2}\left(F^{T} e_{3}\right) \otimes\left(e_{1}^{T} Q^{-1} \mathscr{D}^{-2}\right),\right. \\
& \left.\frac{1}{2}\left(F^{T} e_{3}\right) \otimes\left(e_{2}^{T} Q^{-1} \mathscr{D}^{-2}\right), \frac{1}{2}\left(F^{T} e_{3}\right) \otimes\left(e_{3}^{T} Q^{-1} \mathscr{D}^{-2}\right), 0\right)^{T}, \\
r_{2}= & \left(0,\left(F^{T} e_{3}\right) \otimes\left(e_{1}^{T} \Psi_{5}\right)-D_{11}-D_{22},\left(F^{T} e_{3}\right) \otimes\left(e_{2}^{T} \Psi_{5}\right)-D_{13},\right. \\
& \left.\left(F^{T} e_{3}\right) \otimes\left(e_{3}^{T} \Psi_{5}\right), 0\right)^{T}, \\
r_{3}= & \left(0,\left(F^{T} e_{3}\right) \otimes\left(e_{1}^{T} \Psi_{6}\right),\left(F^{T} e_{3}\right) \otimes\left(e_{2}^{T} \Psi_{6}\right)-D_{21},\right. \\
& \left.\left(F^{T} e_{3}\right) \otimes\left(e_{3}^{T} \Psi_{6}\right)-D_{12}-D_{23}, 0\right)^{T}, \\
r_{4}= & \left(0,\left(e_{1}^{T} \Omega^{-1} B^{3}\right) F^{T} e_{3},\left(e_{2}^{T} \Omega^{-1} B^{3}\right) F^{T} e_{3},\left(e_{3}^{T} \Omega^{-1} B^{3}\right) F^{T} e_{3}, 1\right)^{T}, \\
r_{5}= & \left(\frac{1}{2} Q^{-1} \mathscr{D}^{-1} \pi,-\frac{1}{2}\left(\left(F^{T} e_{3}\right) \otimes\left(e_{1}^{T} Q^{-1} \mathscr{D}^{-2}\right)\right) \pi,\right. \\
& \left.-\frac{1}{2}\left(\left(F^{T} e_{3}\right) \otimes\left(e_{2}^{T} Q^{-1} \mathscr{D}^{-2}\right)\right) \pi,-\frac{1}{2}\left(\left(F^{T} e_{3}\right) \otimes\left(e_{3}^{T} Q^{-1} \mathscr{D}^{-2}\right)\right) \pi, 0\right)^{T},
\end{aligned}
$$

with

$$
\begin{aligned}
& \Psi_{5}=\Omega^{-1}\left(A^{31} D_{11}+A^{31} D_{22}+A^{32} D_{13}\right), \\
& \Psi_{6}=\Omega^{-1}\left(A^{32} D_{21}+A^{33} D_{12}+A^{33} D_{23}\right) .
\end{aligned}
$$

The differences between the eigenvectors for each coordinate direction are subtle, and thus only minor changes are required in the programming. 


\subsection{Non-conservative to conservative transformation}

In order to maintain high-order accuracy in the numerical methods it is necessary to recover from (30) and (32) the eigenvectors of the conservative Jacobian, such that the invariants are expressed in terms of conserved variables. Partial derivatives of non-conservative variables can be expressed in terms of partial derivatives of conserved variables according to $H \equiv \partial W / \partial U$, where

with

$$
\begin{aligned}
& \left(\begin{array}{crr}
-2 I & u \otimes\left(e_{1}^{T} F^{-T}\right) & u \otimes\left(e_{2}^{T} F^{-T}\right)
\end{array}\right. \\
& H=-\frac{1}{2 \rho}\left(\begin{array}{ccc}
0 & \left(F^{T} e_{1}\right) \otimes\left(e_{1}^{T} F^{-T}\right)-2 I & \left(F^{T} e_{1}\right) \otimes\left(e_{2}^{T} F^{-T}\right) \\
0 & \left(F^{T} e_{2}\right) \otimes\left(e_{1}^{T} F^{-T}\right) & \left(F^{T} e_{2}\right) \otimes\left(e_{2}^{T} F^{-T}\right)-2 I \\
0 & \left(F^{T} e_{3}\right) \otimes\left(e_{1}^{T} F^{-T}\right) & \left(F^{T} e_{3}\right) \otimes\left(e_{2}^{T} F^{-T}\right) \\
2 \frac{\mathrm{d} S}{\mathrm{~d} \mathscr{E}} u^{T} & -2 \frac{\mathrm{d} S}{\mathrm{~d} e_{1}^{T} F}-e_{1}^{T} F^{-T} \Psi_{7} & -2 \frac{\mathrm{d} S}{\mathrm{~d} e_{2}^{T} F}-e_{2}^{T} F^{-T} \Psi_{7} \\
u \otimes\left(e_{3}^{T} F^{-T}\right) & 0 \\
\left(F^{T} e_{1}\right) \otimes\left(e_{3}^{T} F^{-T}\right) & 0 \\
\left(F^{T} e_{2}\right) \otimes\left(e_{3}^{T} F^{-T}\right) & 0 \\
\left(F^{T} e_{3}\right) \otimes\left(e_{3}^{T} F^{-T}\right)-2 I & 0 \\
-2 \frac{\mathrm{d} S}{\mathrm{~d} e_{3}^{T} F}-e_{3}^{T} F^{-T} \Psi_{7} & -2 \frac{\mathrm{d} S}{\mathrm{~d} \mathscr{E}}
\end{array}\right),
\end{aligned}
$$

$$
\Psi_{7}=\frac{\mathrm{d} S}{\mathrm{~d} \mathscr{E}}\left(\frac{1}{2}|u|^{2}-\mathscr{E}\right)-\sum_{i, j=1}^{3} \frac{\mathrm{d} S}{\mathrm{~d} F_{i j}} F_{i j} .
$$

The matrices of eigenvectors, $R, L$, of the non-conservative Jacobian $A$ can be transformed to those of the conservative Jacobian $A^{c}=H^{-1} A H$ according to

$$
R^{c}=H^{-1} R, \quad L^{c}=L H .
$$

The inverse of (41), $H^{-1} \equiv \partial U / \partial W$, is given by

$$
H^{-1}=-\rho\left(\begin{array}{ccc}
-I & u \otimes\left(e_{1}^{T} F^{-T}\right) & u \otimes\left(e_{2}^{T} F^{-T}\right) \\
0 & \left(F^{T} e_{1}\right) \otimes\left(e_{1}^{T} F^{-T}\right)-I & \left(F^{T} e_{1}\right) \otimes\left(e_{2}^{T} F^{-T}\right) \\
0 & \left(F^{T} e_{2}\right) \otimes\left(e_{1}^{T} F^{-T}\right) & \left(F^{T} e_{2}\right) \otimes\left(e_{2}^{T} F^{-T}\right)-I \\
0 & \left(F^{T} e_{3}\right) \otimes\left(e_{1}^{T} F^{-T}\right) & \left(F^{T} e_{3}\right) \otimes\left(e_{2}^{T} F^{-T}\right) \\
-u^{T} & \frac{\mathrm{d} \mathscr{E}}{\mathrm{d} e_{1}^{T} F}-e_{1}^{T} F^{-T} E & \frac{\mathrm{d} \mathscr{E}}{\mathrm{d} e_{2}^{T} F}-e_{2}^{T} F^{-T} E \\
u \otimes\left(e_{3}^{T} F^{-T}\right) & 0 \\
\left(F^{T} e_{1}\right) \otimes\left(e_{3}^{T} F^{-T}\right) & 0 \\
\left(F^{T} e_{2}\right) \otimes\left(e_{3}^{T} F^{-T}\right) & 0 \\
\left(F^{T} e_{3}\right) \otimes\left(e_{3}^{T} F^{-T}\right)-I & 0 \\
\frac{\mathrm{d} \mathscr{E}}{\mathrm{d} e_{3}^{T} F}-e_{3}^{T} F^{-T} E & -\frac{\mathrm{d} \mathscr{E}}{\mathrm{d} S}
\end{array}\right) .
$$

\section{CONSTITUTIVE EQUATIONS}

\subsection{Internal energy density}

The internal energy density for a hyperelastic material can be expressed in terms of the invariants $\mathcal{I}_{1}, \mathcal{I}_{2}, \mathcal{I}_{3}$ of any symmetric strain tensor. Formulations of a number of the common strain tensors in terms of $F$ is given in [8]. Taking for example the elastic Green's tensor

$$
C=F^{T} F
$$


then the invariants are

$$
\mathcal{I}_{1}=\operatorname{tr} C, \quad \mathcal{I}_{2}=\frac{1}{2}\left[(\operatorname{tr} C)^{2}-\operatorname{tr} C^{2}\right], \quad \mathcal{I}_{3}=\operatorname{det}|C|,
$$

and the internal energy can be written

$$
\mathscr{E}\left(\mathcal{I}_{1}, \mathcal{I}_{2}, \mathcal{I}_{3}, S\right)=\mathscr{U}\left(\mathcal{I}_{3}, S\right)+\mathscr{W}\left(\mathcal{I}_{1}, \mathcal{I}_{2}, \mathcal{I}_{3}, S\right)
$$

The first term corresponds to a hydrostatic contribution, whilst the second accounts for the elastic energy due to shear strains. In specifying the internal energy in the form of Eq. (47), symmetry of the stress tensor is ensured. In terms of $C$, the Murnaghan formulas Eq. (5) become

$$
\sigma_{i j}=2 \rho C_{i k} \frac{\partial \mathscr{E}}{\partial C_{k j}} .
$$

In this paper, two expressions for the internal energy are considered. The first was presented in [17], and is an isentropic hyperelastic equation of state in terms of the invariants of the elastic Greens tensor

$$
\mathscr{E}\left(\mathcal{I}_{1}, \mathcal{I}_{3}\right)=-\int_{V_{0}}^{V} P(V) \mathrm{d} V+\frac{G}{2 \rho_{0}}\left(\mathcal{I}_{1}-3 \mathcal{I}_{3}^{1 / 3}\right),
$$

where $V=1 / \rho$ is the specific volume, and

$$
P(V)=p_{01}(\eta-1)+p_{02}(\eta-1)^{2}+p_{03}(\eta-1)^{3} \mathrm{GPa}, \quad \eta=V_{0} / V .
$$

Eq. (50) was taken from [29].

The second formulation considered is the isotropic hyperelastic equation of state from [25, 2]

$$
\begin{aligned}
\mathscr{E}\left(\mathcal{I}_{1}, \mathcal{I}_{2}, \mathcal{I}_{3}, S\right)= & \frac{K_{0}}{2 \alpha^{2}}\left(\mathcal{I}_{3}^{\alpha / 2}-1\right)^{2}+c_{v} T_{0} \mathcal{I}_{3}^{\gamma / 2}\left(\exp \left[S / c_{v}\right]-1\right)+ \\
& \frac{B_{0}}{2} \mathcal{I}_{3}^{\beta / 2}\left(\mathcal{I}_{1}^{2} / 3-\mathcal{I}_{2}\right) .
\end{aligned}
$$

where in this case the invariants correspond to the elastic Finger tensor

$$
G=F^{-T} F^{-1} \text {. }
$$

The parameters $K_{0}=c_{0}^{2}-(4 / 3) b_{0}^{2}, B_{0}=b_{0}^{2}$ are the squared bulk speed of sound and the squared speed of shear waves respectively, $c_{v}$ is heat capacity at constant volume, $\alpha, \beta, \gamma$ are constants characterising the nonlinear dependence of sound speeds and temperature on the mass density.

\subsection{Relaxation time of tangential stresses}

One function for the relaxation time capable of modelling sufficiently the physical loading behaviour of metals is the power law function [16]

$$
\tau=\tau_{0}\left(\frac{\sigma_{0}}{\sigma_{I}}\right)^{n}
$$

where $\sigma_{0}, \tau_{0}$ and $n$ are material specific constants, and the shear stress intensity is given by

$$
\sigma_{I}=\sqrt{\left[\left(\sigma_{11}-\sigma_{22}\right)^{2}+\left(\sigma_{22}-\sigma_{33}\right)^{2}+\left(\sigma_{33}-\sigma_{11}\right)^{2}+6\left(\sigma_{12}^{2}+\sigma_{13}^{2}+\sigma_{23}^{2}\right)\right] / 2} .
$$


Determination of the parameters entering into (53) can be determined by performing a series of numerical experiments [15, 22]. Specifically, the system of equations (15) can be reduced for the special case of uniaxial strain of a thin rod (see [8] for a detailed derivation). Simulations can then be performed where the sample is deformed in one coordinate direction at a constant strain rate thus providing stress versus strain data. These numerical tests are analogous to the Split Hopkinson Pressure Bar (SHPB) test [22] commonly employed to investigate high strain rate loading behaviour. Therefore any function minimisation method can be used to determine accurate constants by comparison of the numerical and available experimental data. It is noted that using this technique it is possible to develop models that take into account microscopic phenomena such as dislocation motion, with the necessary constants, not readily available for all metals, being determined via the numerical tests [15, 22]. The model (53) represents a simple empirical model and admits strain rate dependency of the flow stress. Figure 1 illustrates the yield stress against strain rate for different values of $n$. Note that in general the parameters $\sigma_{0}$, $\tau_{0}$ and $n$ will depend on the state of the material [7] to realise influences such as temperature on the yield stress.

In some cases inelastic deformations are required to satisfy idealised plasticity theory, or at least some approximation of it, in order to provide comparison with certain well known numerical experiments of previous studies. That is, the rate of change of stress is equivalently zero under further loading once the stress intensity reaches the material specific yield stress.

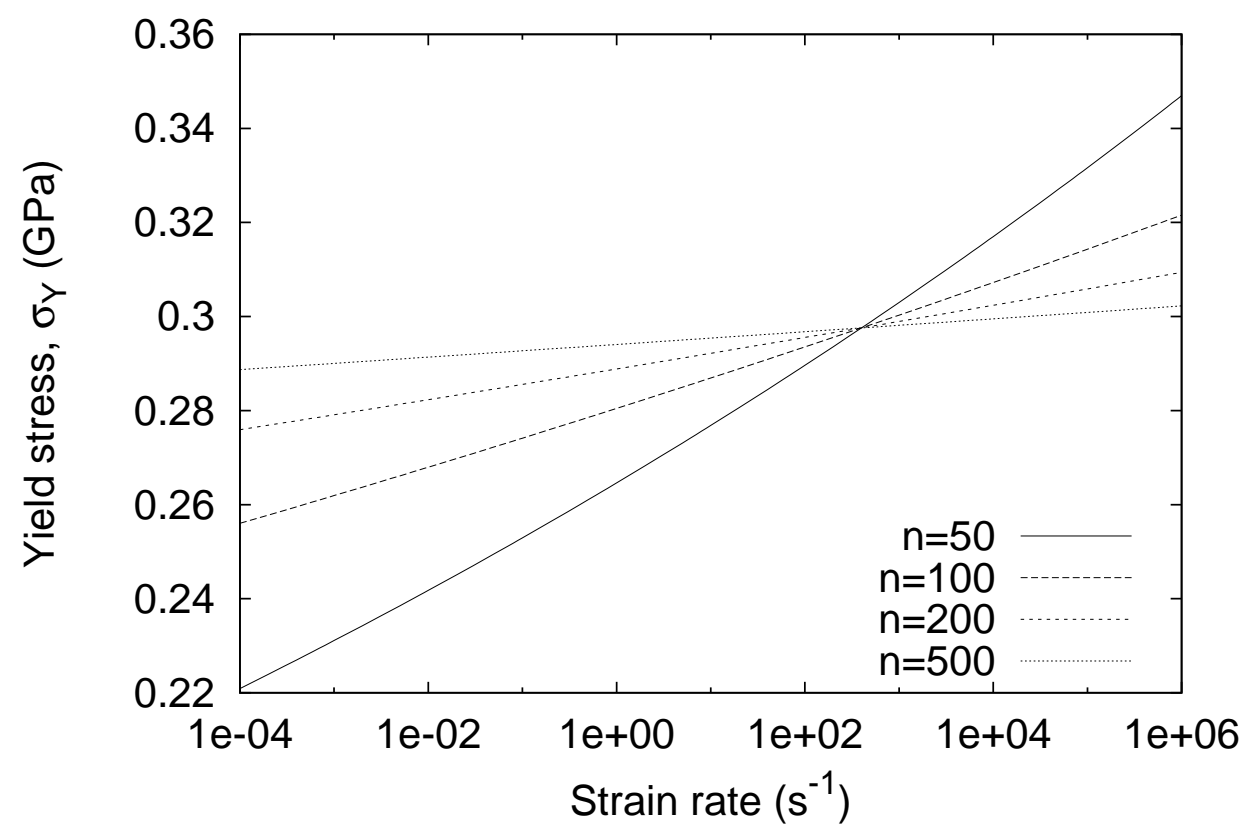

Figure 1. Yield stress plotted against strain rate showing the influence of the parameter $n$ in Eq. (53). Results were obtained using the bar theory in [8], with the isentropic equation of state from $\S 4.1$. The material was aluminium, with constants equal to those in $\S 6.1$. Values of the constants in Eq. (53) were $\sigma_{0}=0.2976 \mathrm{GPa}$ and $\tau_{0}=1 \cdot 10^{-} 5 \mathrm{~s}$. 
Furthermore, the yield surface is required to be independent of strain-rate effects. There are different approaches one can take to achieve ideal plasticity with the present governing model. One would be to use a modified version of the radial return algorithm, see for example $[21,17]$. Another might be to use the modified potential function proposed in [11]. However a different approach is proposed here based upon the model (53). Strain rate effects entering into the governing model vanish in the limit $n \rightarrow \infty$, such that $\sigma \rightarrow \sigma_{0}$ where $\sigma_{0}$ will represent the required yield stress (Figure 1). Of course this can not be achieved in reality, but $n$ can instead be taken large enough such that strain rate effects are decreased. Furthermore in doing so a rigorous test is provided for the time integrator. It is seen (Figure 1) that even at values of $n=500$ strain-rate effects remain apparent, and therefore the model is used for illustrative purposes only, rather than as a suggested method if one requires strict idealised plasticity.

\section{NUMERICAL SCHEME}

The conservative system of equations, Eq. (15), is solved over a fixed, structured computational grid consisting of quadrilateral cells denoted by the indices $i, j, k$. Each cell has the dimensions $\Delta x_{1_{i, j, k}}=x_{1_{i+1 / 2, j, k}}-x_{1_{i-1 / 2, j, k}}, \Delta x_{2_{i, j, k}}=x_{2_{i, j+1 / 2, k}}-x_{2_{i, j-1 / 2, k}}, \Delta x_{3_{i, j, k}}=x_{3_{i, j, k+1 / 2}}-$ $x_{3_{i, j, k-1 / 2}}$, thus forming the control volumes $\mathscr{V}_{i, j, k}=\Delta x_{1_{i, j, k}} \Delta x_{2_{i, j, k}} \Delta x_{3_{i, j, k}}$. Integrating Eq. (15) over $\mathscr{V}_{i, j, k}$ yields the following system of ordinary-differential-equations

$$
\left(\frac{\mathrm{d} U}{\mathrm{~d} t}\right)_{i, j, k}=-\Delta t\left(L_{i, j, k}^{F}+S_{i, j, k}^{p}\right) .
$$

where $U_{i, j, k}$ is the vector of volume averaged conserved variables stored at the cell centres, and the unsplit spatial discretization operator is expressed as

$$
\begin{aligned}
L_{i, j, k}^{F}= & \left\{\frac{\mathscr{F}_{i+1 / 2, j, k}^{1}-\mathscr{F}_{i-1 / 2, j, k}^{1}}{\Delta x_{1_{i, j, k}}}+\frac{\mathscr{F}_{i, j+1 / 2, k}^{2}-\mathscr{F}_{i, j-1 / 2, k}^{2}}{\Delta x_{2_{i, j, k}}}\right. \\
& \left.+\frac{\mathscr{F}_{i, j, k+1 / 2}^{3}-\mathscr{F}_{i, j, k-1 / 2}^{3}}{\Delta x_{3_{i, j, k}}}+S_{i, j, k}^{c}\right\},
\end{aligned}
$$

where $\mathscr{F}_{i \pm 1 / 2, j, k}^{1}, \mathscr{F}_{i, j \pm 1 / 2, k}^{2}, \mathscr{F}_{i, j, k \pm 1 / 2}^{3}$, are the numerical fluxes evaluated at the cell boundaries. To solve the multi-dimensional problem Eqs. (55)-(56) an unsplit dimension-bydimension approach is used. For this, each numerical flux function is calculated via solution of a one-dimensional Riemann problem orientated normal to the respective boundary. For $\mathscr{F}_{i \pm 1 / 2, j, k}^{1}$ then, the solution is found using data in the $i$-direction only. Eq. (56) is therefore constructed using the solutions of six one-dimensional Riemann problems, one across each cell boundary, for each cell in a three dimensional calculation. Only once all the numerical fluxes are summed according to Eq. (56) is the solution advanced in time.

In a recent study by the authors [2] a high-order characteristics based approximate solution of the Riemann problem for the augmented one-dimensional system was presented. This method is outlined in the next section and generalises to all cell boundaries; each case would simply utilise data in the normal direction only and the respective eigenvalues and eigenvectors detailed in $\S 3$. 


\subsection{Evaluation of the convective fluxes}

Consider the non-linear system (21), omitting inelastic source terms, $S^{p}=0$. If it is assumed that the Jacobian $\mathscr{A}$ is evaluated at some constant state $\widehat{W}$ such that $\widehat{A}=A(\widehat{W})$ consists entirely of constant coefficients, then in turn the corresponding eigenvalues and eigenvectors are constant, $\widehat{\Lambda}=\Lambda(\widehat{W}), \widehat{L}=L(\widehat{W}), \widehat{R}=R(\widehat{W})$. If $\mathscr{Q}=\widehat{L} W$ is defined as the vector of characteristic variables, then $(21)$ can be rewritten in the decoupled characteristic form

$$
\left(\frac{\partial}{\partial t}+\widehat{\lambda}_{j} \frac{\partial}{\partial x}\right) \mathscr{Q}_{j}=0
$$

In order to maintain high-order accuracy, the relationships in $\S 3.3$ can be used to reexpress the characteristic variables in terms of conserved variables. Given $\widehat{H}$ evaluated at the constant state, then the characteristic variables are constructed as $\mathscr{Q}_{c}=\widehat{L} \widehat{H} U$. From (57), $\mathscr{Q}_{j}$ is invariant along the characteristic of slope $\widehat{\lambda}_{j}$, thus the solution for any Cauchy problem is simply $\mathscr{Q}_{c_{j}}(x, t)=\mathscr{Q}_{c_{j}}\left(U\left(x-\widehat{\lambda}_{j} t\right)\right)$, which gives

$$
U(x, t)=H^{-1} \widehat{R} \mathscr{Q}_{c}(x, t) .
$$

On a computational mesh these linearised problems are solved at each intercell boundary, $i-1 / 2$. Locally then one is solving exactly an approximation of the non-linear system (15). The constant state $\widehat{W}_{i-1 / 2}$ used to evaluate the coefficients is found via an arithmetic mean of the adjoining left and right cell averaged states

$$
\widehat{W}_{i-1 / 2}=\frac{1}{2}\left(W_{i}+W_{i-1}\right) .
$$

A convenient function that achieves the solution is [5]

$$
\mathscr{Q}\left(x_{i-1 / 2}-\widehat{\lambda}_{j ; i-1 / 2} t\right)=\left(\frac{1}{2}+\psi_{j ; i-1 / 2}\right) \mathscr{Q}_{i-1 / 2}^{L}+\left(\frac{1}{2}-\psi_{j ; i-1 / 2}\right) \mathscr{Q}_{i-1 / 2}^{R},
$$

with

$$
\psi_{j ; i-1 / 2}=\frac{1}{2} \frac{\widehat{\lambda}_{j ; i-1 / 2}}{\left|\widehat{\lambda}_{j ; i-1 / 2}\right|+\varepsilon}, \quad \widehat{\lambda}_{j ; i-1 / 2}=\lambda_{j}\left(\widehat{W}_{i-1 / 2}\right)
$$

where $\mathscr{Q}_{i-1 / 2}^{L}$ and $\mathscr{Q}_{i-1 / 2}^{R}$ represent the left and right characteristic states adjacent to the boundary found by some high-order reconstruction method, and $\varepsilon$ is a small number to prevent division by zero. The found solution (58) can then be used to construct the flux terms in Eq. (56).

High order accuracy is achieved by taking in Eq. (60) boundary-extrapolated values from polynomial reconstruction of cell volume averaged variables. Specifically reconstruction is carried out on characteristic variables using a weighted essentially non-oscillatory (WENO) scheme [9]. In [2] a number of WENO schemes of increasing order were analysed against exact solutions of one-dimensional initial value problems in non-linear elasticity. It was found that high-order schemes are necessary to resolve delicate features such as discontinuities and small amplitude waves. The fifth-order WENO scheme proved to be the most practical for the equations of non-linear elasticity, based upon accuracy and cost. The choice of reconstruction based upon characteristic rather than conserved variables is based on the latter producing in 
many cases severe oscillations across discontinuities. To further eliminate such artifacts the monotonicity preserving modification of [1] is also used.

For a scalar function $\phi(x)$ the fifth order accurate left boundary extrapolated value $\phi_{i-1 / 2}^{L}$ is defined in terms of cell averaged values $\phi_{i}$ as

$$
\begin{gathered}
\phi_{i-1 / 2}^{L}=\frac{1}{6}\left[\omega_{0}\left(-\phi_{i+1}+5 \phi_{i}+2 \phi_{i-1}\right)+\omega_{1}\left(-\phi_{i-2}+5 \phi_{i-1}+2 \phi_{i}\right)+\right. \\
\left.\omega_{2}\left(2 \phi_{i-3}-7 \phi_{i-2}+11 \phi_{i-1}\right)\right]
\end{gathered}
$$

where $\omega_{k}, k=1,2,3$, are nonlinear WENO weights given by

$$
\omega_{k}=\frac{\alpha_{k}}{\sum_{l=0}^{3} \alpha_{l}}, \quad \alpha_{0}=\frac{3}{10\left(\beta_{0}+\varepsilon\right)^{2}}, \quad \alpha_{1}=\frac{3}{5\left(\beta_{1}+\varepsilon\right)^{2}}, \quad \alpha_{2}=\frac{1}{10\left(\beta_{2}+\varepsilon\right)^{2}}
$$

The parameter $\varepsilon$ is introduced to avoid division by zero and the recommended value of $\varepsilon=10^{-6}$ is taken. The smoothness indicators are

$$
\begin{aligned}
& \beta_{1}=(13 / 12)\left(\phi_{i-1}-2 \phi_{i}+\phi_{i+1}\right)^{2}+(1 / 4)\left(3 \phi_{i-1}-4 \phi_{i}+\phi_{i+1}\right)^{2} \\
& \beta_{2}=(13 / 12)\left(\phi_{i-2}-2 \phi_{i-1}+\phi_{i}\right)^{2}+(1 / 4)\left(\phi_{i-2}-\phi_{i}\right)^{2} \\
& \beta_{3}=(13 / 12)\left(\phi_{i-3}-2 \phi_{i-2}+\phi_{i-1}\right)^{2}+(1 / 4)\left(\phi_{i-3}-4 \phi_{i-2}+3 \phi_{i-1}\right)^{2}
\end{aligned}
$$

The right value $\phi_{i-1 / 2}^{R}$ is obtained by symmetry.

\subsection{Evaluation of the compatibility vector}

For each cell, the source term $S^{c}$ is computed according to

$$
S_{i, j, k}^{c}=\left(\begin{array}{c}
0 \\
u_{1_{i, j, k}}^{n} \beta_{i, j, k} \\
u_{2_{i, j, k}}^{n} \beta_{i, j, k} \\
u_{3_{i, j, k}}^{n} \beta_{i, j, k} \\
0
\end{array}\right) .
$$

Here $u_{\alpha_{i, j, k}}^{n}$ denotes the volume averaged velocity components at the centre of cell $i, j, k$, evaluated at the last timestep or sub timestep in the case of multilevel time integration. The vector $\beta$ contains additional derivatives requiring discretization. In the same way as the spatial operator Eq. (56), $\beta$ can be evaluated by taking differences across each cell volume

$$
\begin{aligned}
\beta_{i, j, k}= & \frac{\left(\rho F^{T}\right)_{i+1 / 2} e_{1}-\left(\rho F^{T}\right)_{i-1 / 2} e_{1}}{\Delta x_{1_{i}}}+\frac{\left(\rho F^{T}\right)_{j+1 / 2} e_{2}-\left(\rho F^{T}\right)_{j-1 / 2} e_{2}}{\Delta x_{2_{j}}} \\
& +\frac{\left(\rho F^{T}\right)_{k+1 / 2} e_{3}-\left(\rho F^{T}\right)_{k-1 / 2} e_{3}}{\Delta x_{3_{k}}}
\end{aligned}
$$

Following [17], values of those terms evaluated at the cell boundaries are taken from the high order Riemann problem solution for the corresponding cell edge. In this approach the term $S^{c}$ carries little overhead to the overall scheme; the necessary terms are simply added at the end of each convective flux evaluation. 


\subsection{Time integration}

Devising a numerical scheme to solve Eq. (55) is challenging as a result of the terms in $S^{p}$ governing the onset of inelastic deformations. The relaxation time, $\tau$, of a metal could, under dynamic loading, vary over a large range of order of magnitudes. Consideration of the limits of $\tau$ provides a better understanding of the difficulties, and scope for devising adequate numerical algorithms:

- the elastic limit, $\tau \rightarrow \infty$

- the hydrodynamic limit, $\tau \rightarrow 0$

In the elastic limit, $S^{p} \rightarrow 0$ in Eq. (15), i.e. the system approaches the governing equations of non-linear elasticity. For a solid material the hydrodynamic limit, so called because the material will behave like a fluid, is reached under very high strain rates. In this regime solids can be approximated well by the compressible Euler equations

$$
\frac{\partial \mathcal{U}}{\partial t}+\frac{\partial \mathscr{F}^{\alpha}}{\partial x_{\alpha}}=0
$$

with

$$
\mathcal{U}=\left(\begin{array}{c}
\rho u \\
\rho \\
\rho E
\end{array}\right), \quad \mathscr{F}^{\alpha}=\left(\begin{array}{c}
u_{\alpha} \rho u-p \\
\rho u_{\alpha} \\
u_{\alpha} \rho E-u_{\alpha} p
\end{array}\right),
$$

where $p$ is hydrostatic pressure; and an appropriate equation of state of the form $\mathscr{E}=\mathscr{E}(\rho, S)$.

It is desirable to use for computations a global timestep dictated by the grid sizes and elastic wave speeds

$$
\Delta t=\mathrm{CFL} \times \min _{i, j, k}\left(\frac{\Delta x_{1}}{\left|u_{1}\right|+\mathscr{D}_{\max }^{1}}, \frac{\Delta x_{2}}{\left|u_{2}\right|+\mathscr{D}_{\max }^{2}}, \frac{\Delta x_{3}}{\left|u_{3}\right|+\mathscr{D}_{\max }^{3}}\right),
$$

where $\mathscr{D}_{\max }^{\alpha}$ denotes the maximum wavespeed of the acoustic tensor, Eq. (27), in the direction $\alpha$, and $0 \leq \mathrm{CFL} \leq 1$ is an adjustable scaler parameter used to control the timestep so as to satisfy the Courant-Friedrichs-Lewy (CFL) condition. However based upon the above limits the timescales associated with the relaxation operator can be small in comparison, hence the system of ODEs Eq. (55) will become stiff. Since in most circumstances one cannot forecast zones in which either stiff or non-stiff regimes will be apparent, it is necessary to solve the complete system of equations throughout the domain with an appropriate time integration method that overcomes the problem of stiff ODEs.

Since high order methods have already been established for the homogeneous equations for non-linear elasticity, $S^{p}=0$ in Eq. (15), a natural extension for solving Eq. (55) would be to use temporal splitting

$$
\begin{gathered}
U^{*}=U^{n}-\Delta t L^{F}\left(U^{n}\right), \\
U^{n+1}=U^{*}-\Delta t S^{p}\left(U^{n+1}\right) .
\end{gathered}
$$

That is the homogeneous system, $S^{p}=0$ in Eq. (15), is updated explicitly using the forward Euler method, Eq. (69a), whilst the (possibly stiff) source terms are updated implicitly using Eq. (69b). The latter can then be solved using an $L$-stable implicit integration technique, eliminating any influence of the relaxation time on the CFL condition. Jin [10] discusses the 
use of splitting schemes applied to hyperbolic conservation laws with stiff relaxation operators. Here it is shown that solutions via temporal splitting is sufficient provided that the numerical algorithm has the correct asymptotic limit: in the hydrodynamic limit the scheme is a stable and consistent evaluation of the equilibrium system, Eq. (66), for fixed spatial and temporal step sizes. Violating this property can result in spurious solutions.

To ensure that in the asymptotic limit the numerical method leads to an explicit integration of the equilibrium system, small modifications need to be made to the formulation of governing theory, Eq. (15). As stated in $\S 2$, in conjunction with the equations for $F$ one has the mass continuity equation, (18). As a result, Eq. (18) can be used in place of one equation for the deformation gradient tensor whilst maintaining a fully determined system. One choice would be to replace the equation for $\rho F_{11}$ in (15), thus (16) is replaced with

$$
U=\left(\begin{array}{c}
\rho u \\
\rho \\
\rho F_{12} \\
\vdots \\
\rho F_{33} \\
\rho E
\end{array}\right), \quad \mathscr{F}^{\alpha}=\left(\begin{array}{c}
u_{\alpha} \rho u-e_{\alpha}^{T} \sigma \\
\rho u_{\alpha} \\
\rho u_{\alpha} F_{12}-\rho u_{1} F_{\alpha 2} \\
\vdots \\
\rho u_{\alpha} F_{33}-\rho u_{3} F_{\alpha 3} \\
u_{\alpha} \rho E-u e_{\alpha}^{T} \sigma
\end{array}\right)
$$

and the source terms (17) adjusted accordingly for the zero right-hand side of (18). Eq. (4) can be rearranged to recover $F_{11}$ in terms of $\rho_{0}, \rho, F_{12}, \ldots, F_{33}$. Following [4], there now exists a constant $5 \times 13$ matrix

$$
\mathcal{Q}=\left(\begin{array}{lllllllllllll}
1 & 0 & 0 & 0 & 0 & 0 & 0 & 0 & 0 & 0 & 0 & 0 & 0 \\
0 & 1 & 0 & 0 & 0 & 0 & 0 & 0 & 0 & 0 & 0 & 0 & 0 \\
0 & 0 & 1 & 0 & 0 & 0 & 0 & 0 & 0 & 0 & 0 & 0 & 0 \\
0 & 0 & 0 & 1 & 0 & 0 & 0 & 0 & 0 & 0 & 0 & 0 & 0 \\
0 & 0 & 0 & 0 & 0 & 0 & 0 & 0 & 0 & 0 & 0 & 0 & 1
\end{array}\right)
$$

that satisfies $\mathcal{Q} S^{p}=0$ and gives $\mathcal{U}=\mathcal{Q} U$, i.e. recovers the conserved variables of the Euler equations. Furthermore it is assumed that $S^{p}(U)=0$ can be solved uniquely in terms of $\mathcal{U}$ : assuming a function $\mathcal{E}$, giving $U=\mathcal{E}(\mathcal{U})$, then $S^{p}(\mathcal{E}(\mathcal{U}))=0$ [4]. This is apparent since for the Euler equations the devitoric stress tensor is zero, thus giving the required result. Using these results the analysis in $\oint 3.2$ of [20] can be applied to Eqs. (69a)-(69b), to prove that in the limit $\tau \rightarrow 0$ the scheme is an explicit evaluation of the compressible Euler equations.

The accuracy of the explicit update, (69a), has been improved by instead using the third order TVD Runge-Kutta method [24]

$$
\begin{aligned}
U^{(1)} & =U^{n}+\Delta t L^{F, n}, \\
U^{(2)} & =U^{n}+\frac{1}{4} \Delta t L^{F,(1)}+\frac{1}{4} \Delta t L^{F, n}, \\
U^{*} & =U^{n}+\frac{1}{6} \Delta t L^{F, n}+\frac{1}{6} \Delta t L^{F,(1)}+\frac{2}{3} \Delta t L^{F,(2)} .
\end{aligned}
$$

This is consistent with the TVD requirements of the WENO reconstruction scheme used in the evaluation of convective flux terms. Since stress can be expressed as functions of the state parameters the implicit part (69a) is solved using the iterative Newton-Raphson method. 


\section{1. one-dimensional testcases}

\section{EXAMPLES}

For a test in one-dimension the plate impact problem of Wilkins [29] is considered. The problem consists of an $0.5 \mathrm{~cm}$ aluminium flyer plate impacting a semi-infinite aluminium target. These tests were conducted using a similar Eulerian model in [17], where they proposed the isentropic hyperelastic equation of state for internal energy discussed in $\S 4.1$. In both $[29,17]$ the material is assumed to obey ideal plasticity. This is approximated here using the model Eq. (53). Constants used for the constitutive equations correspond to Aluminium: $G=24.8 \mathrm{GPa}, \rho_{0}=2.7 \mathrm{~kg} \mathrm{~m}^{-3}, p_{01}=73 \mathrm{GPa}, p_{02}=172 \mathrm{GPa}, p_{03}=40 \mathrm{GPa}, \sigma_{0}=0.2976 \mathrm{GPa}$, $n=100, \tau_{0}=1$.

Two impact velocities are investigated, $u_{1}=0.8 \mathrm{~km} \mathrm{~s}^{-1}$ and $u_{1}=2.0 \mathrm{~km} \mathrm{~s}^{-1}$. In both cases the grid consists of 500 cells in the interval $[0: 5] \mathrm{cm}$, with the interface initially located at $x_{0}=0.5 \mathrm{~cm}$, and $\mathrm{CFL}=0.6$. The simulation starts with the flyer plate in contact with the target.

The numerical algorithm for this problem was modified to accommodate the unconstrained free boundary of the flyer plate. A detailed description of these algorithms shall be left to a later paper and only a brief summary is given here. The interface location is tracked via a levelset field which identifies those cells within the material and designates cells in the void region as ghost cells whose state is determined via solution of a Riemann problem [14]. In this case a solid-vacuum Riemann problem is solved using a method similar to that proposed in [18]. It is mentioned that a level-set method is by no means a definitive approach for extending the proposed single component scheme for modelling multi-components. Similar models to those employed in the current paper have been combined with volume-of-fluid (VOF) methods [18], marker particle methods [27] and moving grid methods [26]. A level-set method has been chosen since, unlike conventional VOF methods, this has the potential to allow sliding between components, and does not posses the complexity of marker particle methods when extending to multi-dimensions. Level-set methods do however suffer from spurious mass loss as a result of the inherent regularisation of the numerical scheme used to discretize spatial derivatives when advecting the level-set fields. In the one-dimensional testcases here, with the high grid resolutions employed and use of the fifth-order numerical method from [19] to discretize spatial derivatives in the level-set convection equation, these conservation errors are reduced to negligible amounts. However, this will not necessarily be the case in multi-dimensional cases and further efforts may be required in such circumstances to combat conservation errors.

For the lower of the two impact velocities the time series in Figures 2-3 shows that the impact results in shock waves propagating into both the flyer plate and the target. The left travelling wave eventually reaches the free surface and is subsequently reflected resulting in a right travelling rarefaction wave. At later times elastic plastic flow is clearly distinguishable from the characteristic splitting of waves; the so called elastic precursor travelling faster than the plastic wave. Unfortunately no analytic solution exists for this problem, but both the wave speeds and the respective jump in properties are in good qualitative agreement with $[29,17]$. It is noted that taking $n=100$ in Eq. (53) does not provide strain-rate independent flow stress (Figure 1), and indeed it can be expected that strain rate effects manifest within the results, hence no more than a qualitative analysis could be made regardless of the availability of analytic solutions for idealised plasticity. 
Despite this, the use of the Eq. (53) provides a rigorous test on the robustness of the time integration scheme since the large power $n$ results in sharp changes between non-stiff and stiff regimes. Furthermore the form of Eq. (53) is representative of the types of models one would like to employ for obtaining physically realistic solutions. The higher velocity impact further illustrates the robustness of the numerical techniques. The higher peak stresses mean that the plastic wave travels faster and no splitting of the elastic and plastic parts can be distinguished (Figures 4-5). These behaviours indicate that the hydrodynamic limit is approached, yet the scheme predicts correct wavespeeds and high wave resolution using underresolved discretization parameters.

\section{2. two-dimensional testcase}

For a two-dimensional example consider a plane of material, with the origin located at $\left(x_{1}=0, x_{2}=0\right)$. The region $r>2 \mathrm{~cm}$ (where $\left.r=\sqrt{x_{1}^{2}+x_{2}^{2}}\right)$ is raised to a pressure $p=10 \mathrm{GPa}$ (where $p=-\rho^{2}(\partial \mathscr{U} / \partial \rho)$ ) and temperature $T=600 \mathrm{~K}$, whilst the inner cylinder, $r \leq 2 \mathrm{~cm}$, is initiated at ambient conditions, $F=I, T=300 \mathrm{~K}$. Both regions are initially at rest. The test is chosen on the basis that the resulting behaviours should be cylindrically symmetric, thus allowing comparison with one-dimensional polar calculations. The hyperelastic equation of state from [25], detailed in $\S 4.1$ is used, with the material constants taken for copper: $\rho_{0}=8.9 \mathrm{~g} \mathrm{~cm}^{-3}, c_{0}=4.651 \mathrm{~km} \mathrm{~s}^{-1}, b_{0}=2.141 \mathrm{kms}^{-1}, c_{v}=3.9 \cdot 10^{-4} \mathrm{~kJ} \mathrm{~g}^{-1} \mathrm{~K}^{-1}, T_{0}=300 \mathrm{~K}$, $\alpha=1.0, \beta=3.0, \gamma=2.0$. For the relaxation model, Eq. (53) is used with the following constants for copper taken from [16]: $\tau_{0}=0.92 \mathrm{~s}, \sigma_{0}=0.045 \mathrm{GPa}, n=10.1$. These were found in [16] on the basis of producing a best match with experimental data. Solutions were found using a computational domain $[0: 10,0: 10] \mathrm{cm}$, where the axis are modelled as symmetric boundaries. The grid was uniform, with $\Delta x_{1}=\Delta x_{2}=1 / 25 \mathrm{~cm}$, and the timestep was restricted using $\mathrm{CFL}=0.6$. For comparison, one-dimensional cylindrical polar results were obtained (see Appendix), using $\Delta r=1 / 50 \mathrm{~cm}$ and $\mathrm{CFL}=0.6$.

The initial conditions result in a cylindrical shock converging on the origin through the central ambient material, which subsequently reflects from the origin. Inspired by the presentation of cylindrically symmetric examples in [17], the Cartesian results are plotted as a scatter graph in Figure 6 so as to examine the extent to which cylindrical symmetry is preserved. Results are shown at time $t=10 \mu \mathrm{s}$, after the shock has reflected from the origin. Overall agreement is good for both the wavespeeds and profiles. The preservation of cylindrical symmetry is also good.

\section{3. three-dimensional testcase}

For a three dimensional testcase initial conditions are taken similar to the previous twodimensional case. Consider a volume of material, with the origin ocated at $\left(x_{1}=0, x_{2}=\right.$ $0, x_{3}=0$ ). The region $r>2 \mathrm{~cm}$ (where $r=\sqrt{x_{1}^{2}+x_{2}^{2}+x_{3}^{2}}$ ), is raised to a pressure $p=10 \mathrm{GPa}$ and temperature $T=600 \mathrm{~K}$, whilst the inner sphere, $r \leq 2 \mathrm{~cm}$, is initiated at ambient conditions, $F=I, T=300 \mathrm{~K}$. Again both regions are initially at rest. The material is again assumed to be copper, with the constitutive equations and respective constants equal to those before. Solutions were found using a computational domain $[0: 10,0: 10,0: 10] \mathrm{cm}$, where the $x_{1}=0, x_{2}=0, x_{3}=0$ planes were modelled as symmetric boundaries. The grid was uniform, with $\Delta x_{1}=\Delta x_{2}=\Delta x_{3}=1 / 10 \mathrm{~cm}$, and the timestep was restricted using $\mathrm{CFL}=0.6$. Similarly, one-dimensional spherical polar results were obtained for reference (see Appendix), 

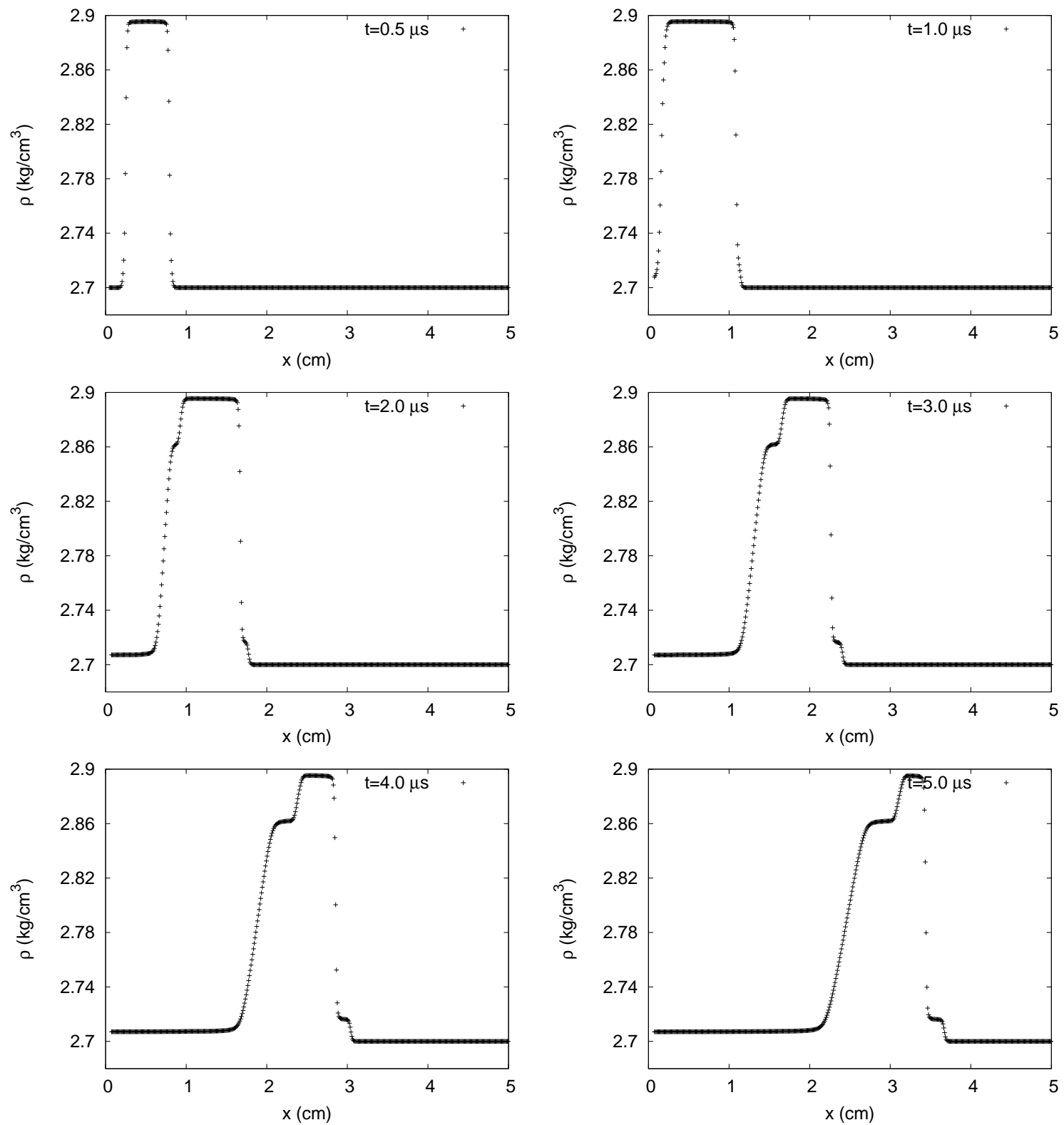

Figure 2. Time sequence of density profiles for an aluminium flyer plate impacting an aluminium target at $0.8 \mathrm{~km} \mathrm{~s}^{-1}(\S 6.1)$. Results were obtained with a uniform grid spacing $\Delta x=1 / 100 \mathrm{~cm}$ and using $\mathrm{CFL}=0.6$. 

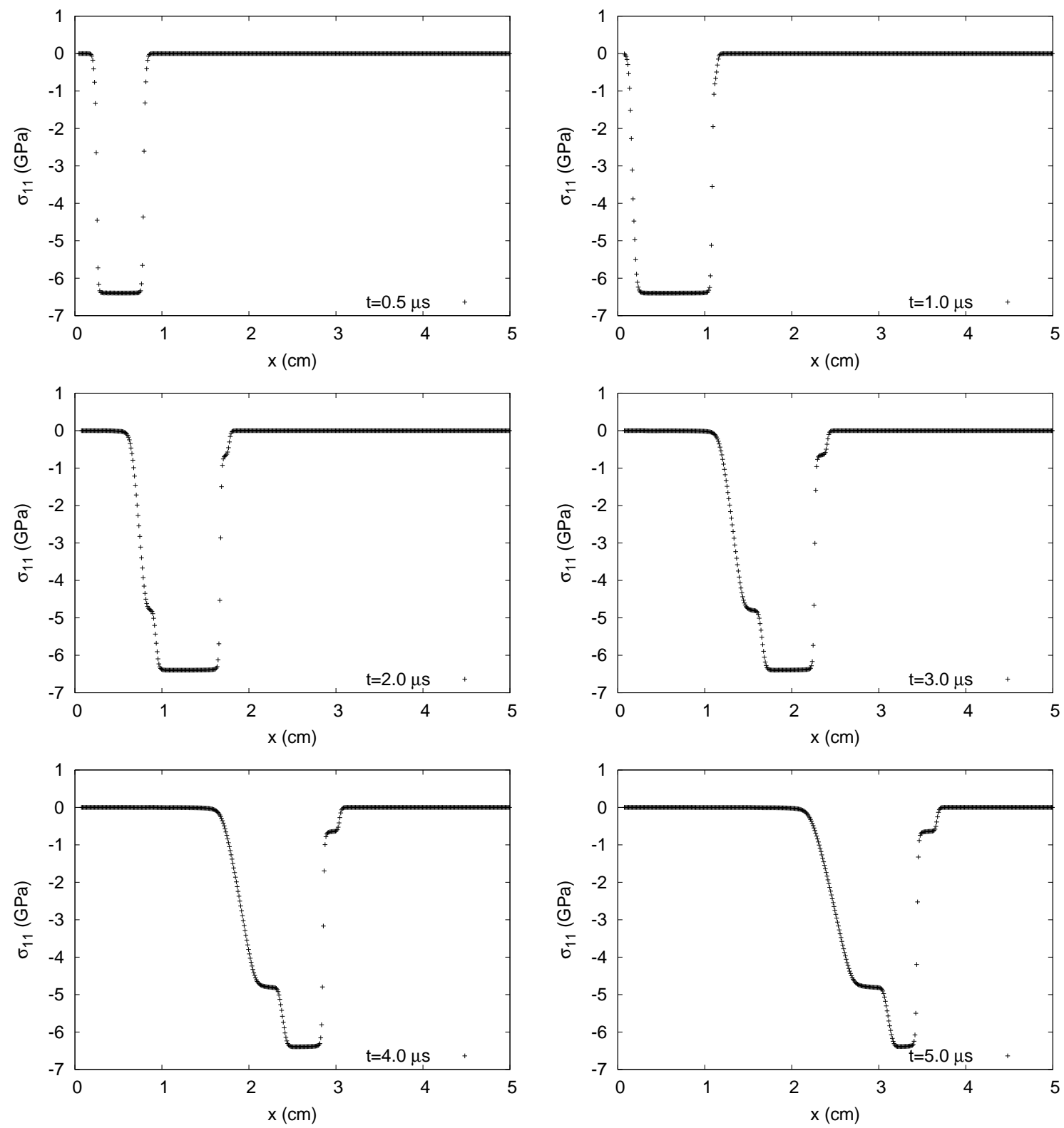

Figure 3. Time sequence of normal stress profiles for an aluminium flyer plate impacting an aluminium target at $0.8 \mathrm{~km} \mathrm{~s}^{-1}(\S 6.1)$. Results were obtained with a uniform grid spacing $\Delta x=1 / 100 \mathrm{~cm}$ and using $\mathrm{CFL}=0.6$. 

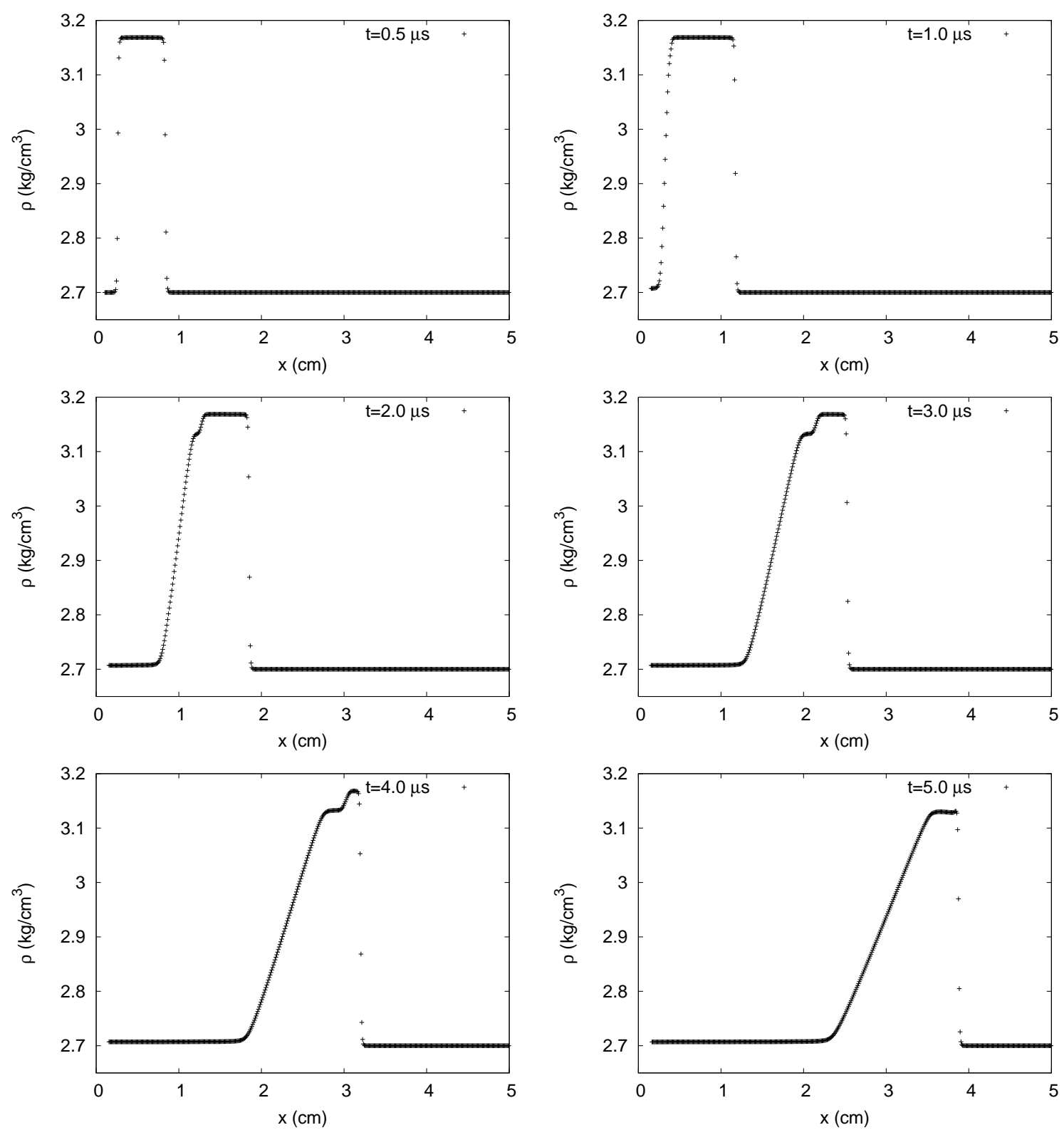

Figure 4. Time sequence of density profiles for an aluminium flyer plate impacting an aluminium target at $2.0 \mathrm{~km} \mathrm{~s}^{-1}(\S 6.1)$. Results were obtained with a uniform grid spacing $\Delta x=1 / 100 \mathrm{~cm}$ and using $\mathrm{CFL}=0.6$. 

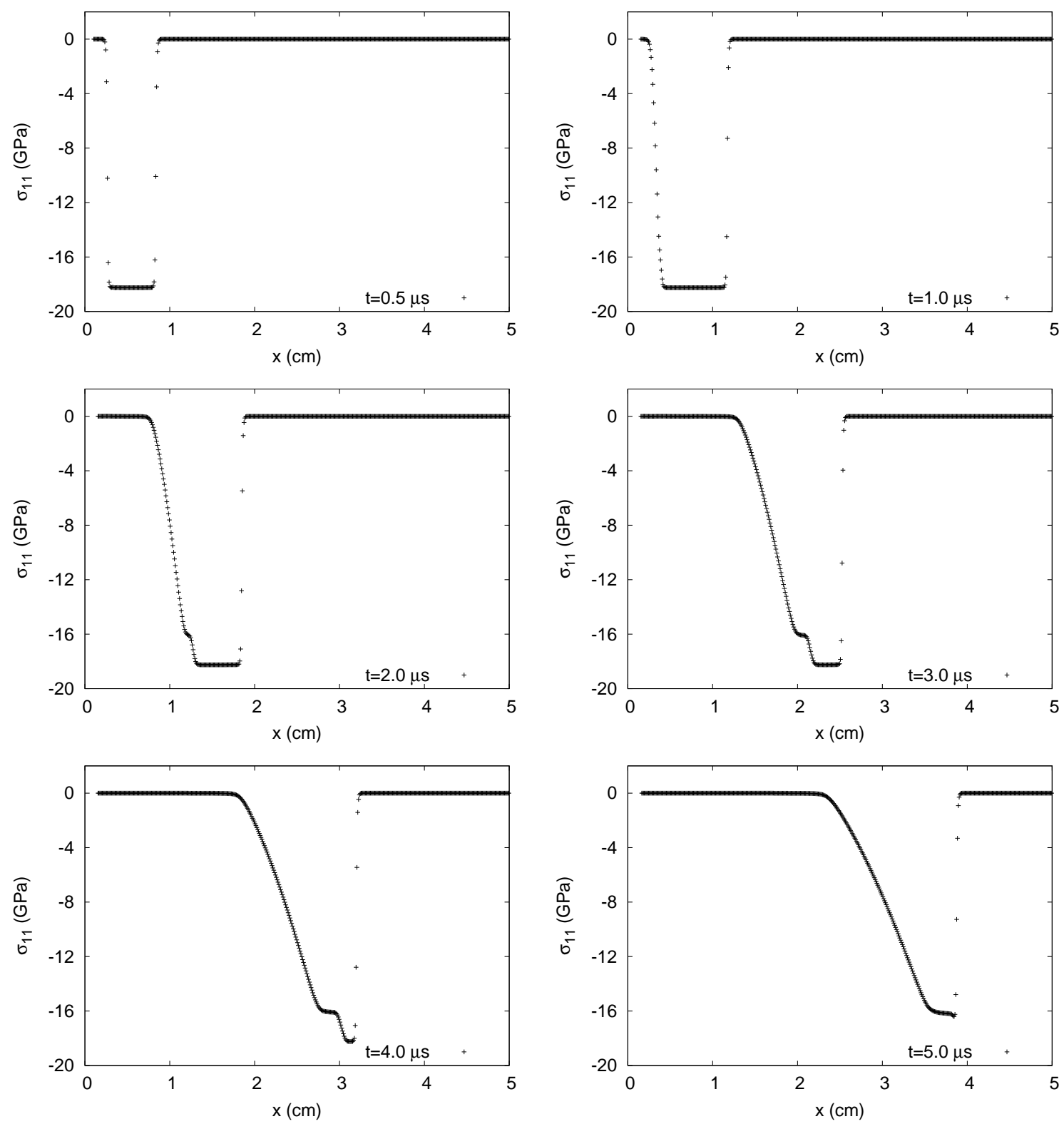

Figure 5. Time sequence of normal stress profiles for an aluminium flyer plate impacting an aluminium target at $2.0 \mathrm{~km} \mathrm{~s}^{-1}(\S 6.1)$. Results were obtained with a uniform grid spacing $\Delta x=1 / 100 \mathrm{~cm}$ and using $\mathrm{CFL}=0.6$. 

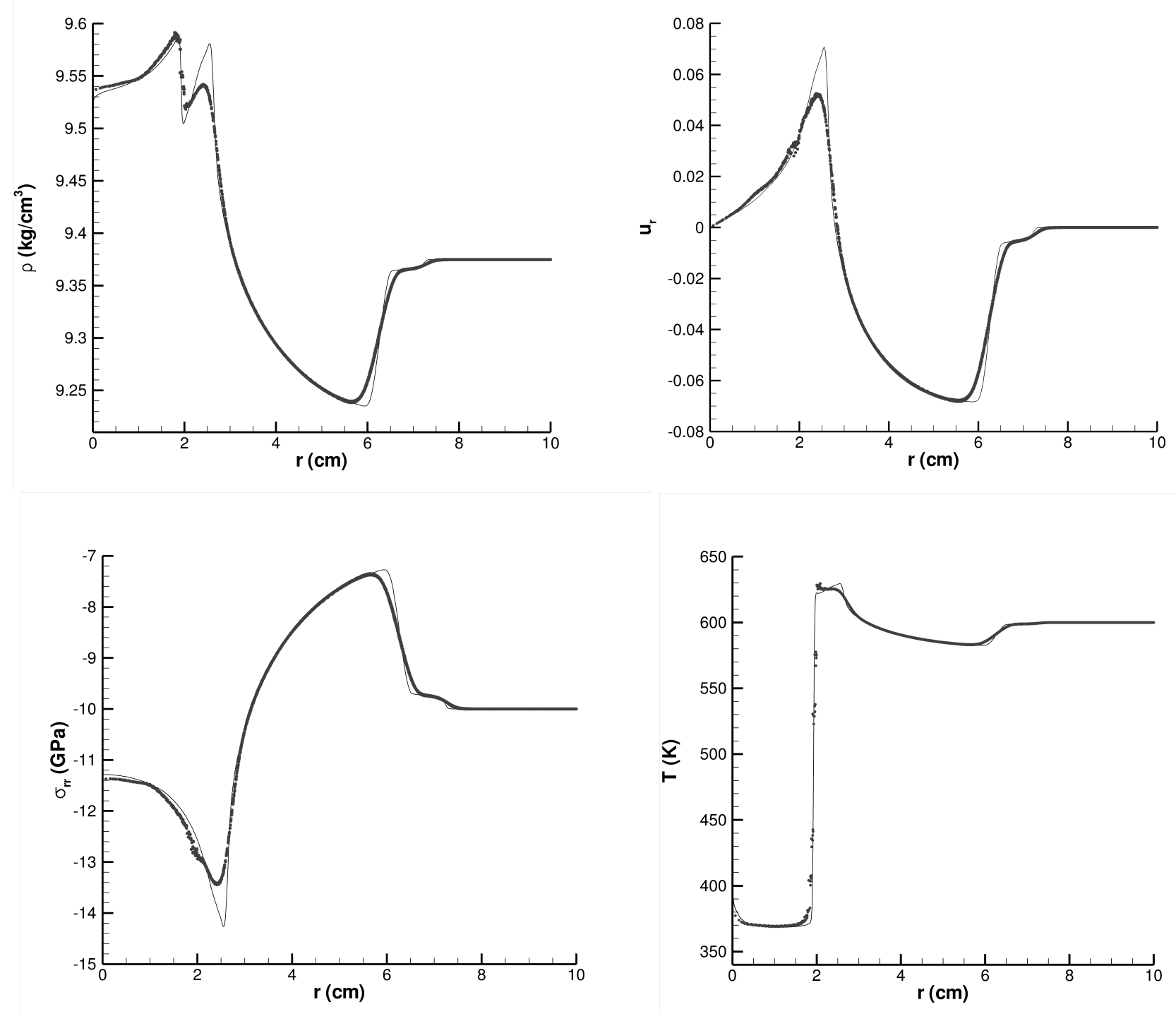

Figure 6. Scatter graph comparison of two-dimensional cartesian (points) and one-dimensional cylindrical (solid line) solutions of the testcase in $\S 6.2$ at a time $t=10 \mu \mathrm{s}$. Cartesian results were obtained with a uniform grid spacing $\Delta x=\Delta y=1 / 25 \mathrm{~cm}$ and using $\mathrm{CFL}=0.6$. 
using $\Delta r=1 / 50 \mathrm{~cm}$ and $\mathrm{CFL}=0.6$.

Both the wavespeeds and profiles are again in good agreement with the one-dimensional results (Figure 7), despite the lower resolution. Spherical symmetry is also seen to be preserved to a good degree.

\section{CONCLUSIONS}

In this paper a high-order shock capturing method was proposed for simulating elastoplastically deforming solid media. The methods were based upon a governing model cast in the Eulerian reference frame, thus allowing the use of fixed Cartesian grids. In a previous study by the authors the reduced system of non-linear elasticity was investigated and high-order approximations of the Riemann problem for the augmented one-dimensional system were developed and tested. The extension of these to three-dimensions proved straightforward requiring only small modifications to the eigenstructure to avoid divisions by zero. The numerical tools employed are well established in other areas of physics, predominantly fluid mechanics. The extension to model plasticity required additional terms to be evaluated. These pose a difficulty to the existing algorithm since the relaxation terms can vary from zero to infinity. However analysis of the model revealed that in the latter limit the system can be approximated well by the simpler compressible Euler equations, and that a simple reformulation yields a mapping between both systems. Application of straightforward temporal splitting is valid in this case as a result of these modifications. Thus the existing methods developed for the homogeneous reduced system of non-linear elasticity needed no further modification and are solved as before using explicit time integration. The addition therefore due to plasticity is a subsequent time integration of the relaxation terms. In this second step the relaxation terms are updated implicitly to overcome numerical stiffness when the relaxation time becomes small in comparison to the employed global timestep dictated by the characteristic speeds.

Simple functions for the relaxation time were taken that result in strain-rate dependency of the flow stress. The form chosen is representative of the types of models that can be used to obtain physically realistic results. In addition, the particular form, a power law function, provided a rigorous test of the time integration methods. This strain on the methods was further amplified by using the model to approximate ideal plasticity. Using this assumption onedimensional test were conducted simulating plate impact experiments. The processes involved moderate to high strain-rates. In the numerical calculations the discretization parameters were underresolved, yet the scheme successfully provided results qualitatively comparable to other results reported in the litrature. The scheme was also applied to examples in two- and threedimensions. In these cases the constants entering into the constitutive equations were taken from the litrature where they were derived based upon best match with experimental data. The tests were cylindrically and spherically symmetric respectively and in both cases symmetry was preserved by the Cartesian solver when compared to one-dimensional polar calculations.

\section{ACKNOWLEDGEMENTS}

The financial support from the Engineering and Physical Sciences Research Council (EP/D051940/1) 

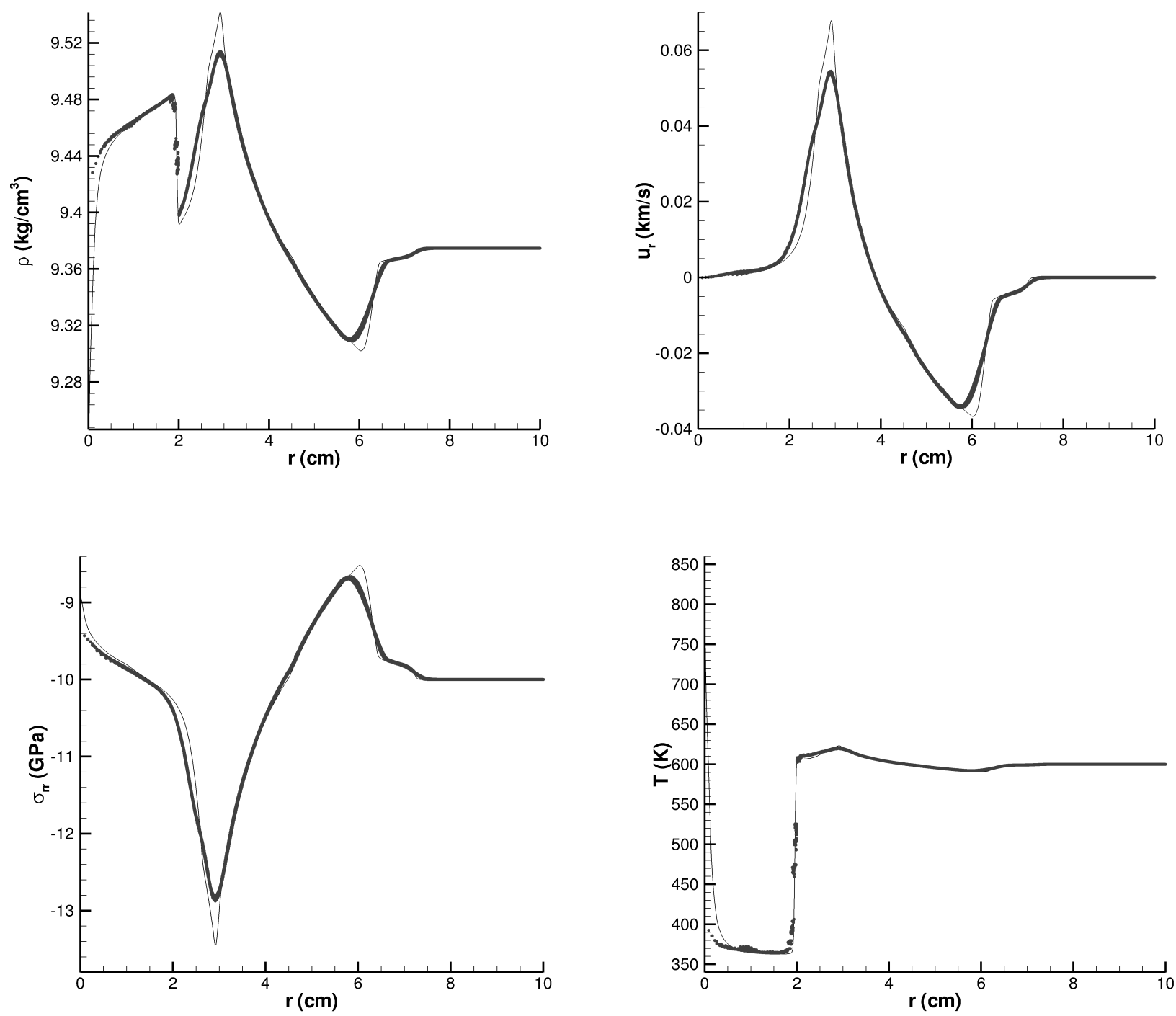

Figure 7. Scatter graph comparison of three-dimensional cartesian (points) and one-dimensional spherical (solid line) solutions of the testcase in $\S 6.3$ at a time $t=10 \mu \mathrm{s}$. Cartesian results were obtained with a uniform grid spacing $\Delta x=\Delta y=\Delta z=1 / 10 \mathrm{~cm}$ and using $\mathrm{CFL}=0.6$. 
and the MoD-AWE Joint Grant Scheme (JGS 607) is greatly appreciated. The first author thanks Dr Evgeniy Shapiro for assistance with the coding.

\section{APPENDIX}

\section{PROOF OF CONTINUITY}

In quasi-linear form the equations governing strain are

$$
\frac{\partial F_{i j}}{\partial t}+u_{k} \frac{\partial F_{i j}}{\partial x_{k}}-F_{k j} \frac{\partial u_{i}}{\partial x_{k}}=-\frac{1}{2 G \tau} \sigma_{i m}^{\prime} F_{m j}
$$

Following [8] the following equalities can be found

$$
\begin{aligned}
D_{t} \rho=\frac{\partial \rho}{\partial F_{i j}} D_{t} F_{i j} & \equiv \frac{\partial \rho}{\partial F_{i j}}\left(\frac{\partial F_{i j}}{\partial t}+u_{k} \frac{\partial F_{i j}}{\partial x_{k}}\right) \\
& =\frac{\partial \rho}{\partial F_{i j}}\left(F_{k j} \frac{\partial u_{i}}{\partial x_{k}}-\frac{1}{2 G \tau} \sigma_{i m}^{\prime} F_{m j}\right)
\end{aligned}
$$

where $D_{t} \equiv \frac{\partial}{\partial t}+u_{k} \frac{\partial}{\partial x_{k}}$ denotes the substantial derivative. Using $\rho=\rho_{0} / \operatorname{det}|F|$, the derivatives of density with respect to deformation in (74) are

$$
\begin{aligned}
\frac{\partial \rho}{\partial F_{i j}} & =-\frac{\rho_{0}}{\operatorname{det}|F|^{2}} \frac{\partial \operatorname{det}|F|}{\partial F_{i j}} \\
& =-\frac{\rho_{0}}{\operatorname{det}|F|} F_{i j}^{-T}=-\rho F_{i j}^{-T} .
\end{aligned}
$$

Thus

$$
\begin{aligned}
\frac{\partial \rho}{\partial F_{i j}} F_{k j} \frac{\partial u_{i}}{\partial x_{k}} & =-\rho F_{i j}^{-T} F_{j k}^{T} \frac{\partial u_{i}}{\partial x_{k}} \\
& =-\delta_{i k} \frac{\partial u_{i}}{\partial x_{k}}=-\rho \frac{\partial u_{k}}{\partial x_{k}}
\end{aligned}
$$

Expanding the continuity equation gives

$$
\begin{aligned}
\frac{\partial \rho}{\partial t}+u_{k} \frac{\partial \rho}{\partial x_{k}}+\rho \frac{\partial u_{k}}{\partial x_{k}} & =\frac{\partial \rho}{\partial F_{i j}}\left(\frac{\partial F_{i j}}{\partial t}+u_{k} \frac{\partial F_{i j}}{\partial x_{k}}-F_{k j} \frac{\partial u_{i}}{\partial x_{k}}\right) \\
& =\frac{\partial \rho}{\partial F_{i j}}\left(-\frac{1}{2 G \tau} \sigma_{i m}^{\prime} F_{m j}\right) .
\end{aligned}
$$

Finally, the previous results can be used to find

$$
\begin{aligned}
\frac{\partial \rho}{\partial F_{i j}}\left(-\frac{1}{2 G \tau} \sigma_{i m}^{\prime} F_{m j}\right) & =\frac{\rho}{2 G \tau} F_{i j}^{-T} F_{j m}^{T} \sigma_{i m}^{\prime} \\
& =\frac{\rho}{2 G \tau} \delta_{i m} \sigma_{i m}^{\prime}=0,
\end{aligned}
$$

Thus the right-hand-side of (77) is equal to zero, which is the required result. 


\section{CYLINDRICAL COORDINATES}

Partial derivatives in Cartesian coordinates, $(x, y, z)$, can be transformed into cylindrical polar coordinates, $(r, \theta, z)$, using

$$
\begin{aligned}
& x=r \cos \theta \\
& y=r \sin \theta \\
& z=z
\end{aligned}
$$

Vectors and tensors are rotated via the rotation matrix

$$
R_{c y l}=\left(\begin{array}{ccc}
\cos \theta & \sin \theta & 0 \\
-\sin \theta & \cos \theta & 0 \\
0 & 0 & 1
\end{array}\right)
$$

giving for example the velocity vector $v_{c y l}=R_{c y l} v$ and deformation tensor $F_{c y l}=R_{c y l} F R_{c y l}$ [17]. Thus the governing system, (15), in cylindrical coordinates becomes

$$
\frac{\partial U}{\partial t}+\frac{1}{r} \frac{\partial r \mathscr{F}^{r}}{\partial r}+\frac{1}{r} \frac{\partial \mathscr{F}^{\theta}}{\partial \theta}+\frac{\partial \mathscr{F}^{z}}{\partial z}=S^{g}-S^{c}-S^{p}
$$

where the flux vectors $\mathscr{F}^{\alpha}$, and inelastic source terms $S^{p}$ maintain the same form as before only the state variables are replaced with those rotated into the new coordinate system. The source term $S^{c}$ and resultant geometric source terms $S^{g}$ are given by

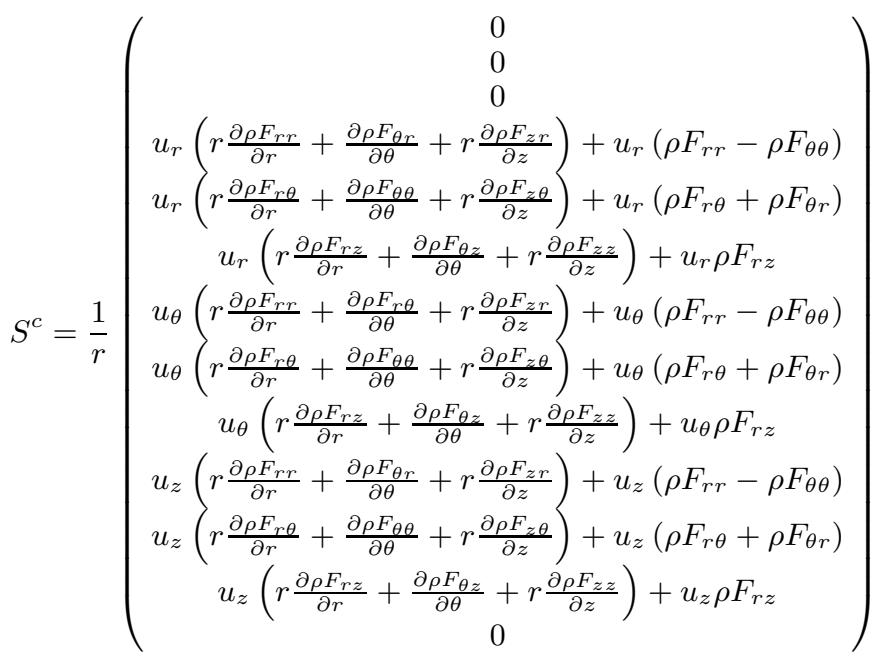

$$
\begin{aligned}
& S^{g}=\frac{1}{r}\left(\begin{array}{c}
\rho u_{\theta}^{2}-\sigma_{\theta \theta} \\
-\rho u_{r} u_{\theta}+\sigma_{r \theta} \\
0 \\
\rho F_{r \theta} u_{\theta}-\rho F_{\theta \theta} u_{r} \\
\rho F_{\theta r} u_{r}-\rho F_{r r} u_{\theta} \\
0 \\
\rho F_{\theta r} u_{r}-\rho F_{r r} u_{\theta} \\
\rho F_{\theta \theta} u_{r}-\rho F_{r \theta} u_{\theta} \\
\rho F_{\theta z} u_{r}-\rho F_{r z} u_{\theta} \\
\rho F_{z \theta} u_{\theta}-\rho F_{\theta \theta} u_{z} \\
\rho F_{\theta r} u_{z}-\rho F_{z r} u_{\theta} \\
0 \\
0
\end{array}\right)
\end{aligned}
$$


Partial derivatives in Cartesian coordinates $(x, y, z)$ can be transformed into spherical polar coordinates, $(r, \theta, \phi)$, using

$$
\begin{aligned}
& x=r \sin \theta \cos \phi \\
& y=r \sin \theta \sin \phi \\
& z=r \cos \theta
\end{aligned}
$$

Vectors and tensors are rotated in the same way as with the cylindrical coordinate transformation, via the rotation matrix

$$
R_{s p h}=\left(\begin{array}{ccc}
\sin \theta \cos \phi & \cos \theta \cos \phi & -\sin \phi \\
\sin \theta \sin \phi & \cos \theta \sin \phi & \cos \phi \\
\cos \theta & -\sin \theta & 0
\end{array}\right)
$$

Thus the system of conservation laws, (15) can be rewritten in spherical coordinates

$$
\frac{\partial U}{\partial t}+\frac{1}{r^{2}} \frac{\partial r^{2} \mathscr{F}^{r}}{\partial r}+\frac{1}{r \sin \theta} \frac{\partial \sin \theta \mathscr{F}^{\theta}}{\partial \theta}+\frac{1}{r \sin \theta} \frac{\partial \mathscr{F}^{\phi}}{\partial \phi}=S^{g}-S^{c}-S^{p}
$$

where the vectors are given by

$$
S^{g}=\frac{1}{r}\left(\begin{array}{c}
\rho u_{\theta}^{2}-\sigma_{\theta \theta}+\rho u_{\phi}^{2}-\sigma_{\phi \phi} \\
-\rho u_{r} u_{\theta}+\sigma_{\theta r}+\cot \theta\left(\rho u_{\phi}^{2}-\sigma_{\phi \phi}\right) \\
-\cot \theta\left(\rho u_{\phi} u_{\theta}-\sigma_{\theta \phi}\right)-\rho u_{\phi} u_{r}+\sigma_{r \phi} \\
\rho u_{\theta} F_{r \theta}-\rho u_{r} F_{\theta \theta}-\rho u_{r} F_{\phi \phi}+\rho u_{\phi} F_{r \phi} \\
\rho F_{\theta r} u_{r}-\rho F_{r r} u_{\theta}-\cot \theta\left(\rho u_{r} F_{\phi \phi}-\rho u_{\phi} F_{r \phi}\right) \\
\cot \theta\left(\rho u_{r} F_{\phi \theta}-\rho u_{\phi} F_{r \theta}\right)+\rho u_{r} F_{\phi r}-\rho u_{\phi} F_{r r} \\
\rho u_{r} F_{\theta r}-\rho u_{\theta} F_{r r}-\rho u_{\theta} F_{\phi \phi}+\rho u_{\phi} F_{\theta \phi} \\
\left.\rho u_{r} F_{\theta \theta}+\rho u_{\theta} F_{r \theta}+\cot \theta-\rho u_{\theta} F_{\phi \phi}+\rho u_{\phi} F_{\theta \phi}\right) \\
\rho u_{r} F_{\theta \phi}+\rho u_{\theta} F_{r \phi}+\cot \theta\left(\rho u_{r} F_{\phi \theta}-\rho u_{\phi} F_{\theta \theta}\right)+\rho u_{\theta} F_{\phi r}-\rho u_{\phi} F_{\theta r} \\
\rho u_{\theta} F_{\phi \theta}-\rho u_{\phi} F_{\theta \theta}+\cot \theta\left(\rho u_{\theta} F_{\phi r}-\rho u_{\phi} F_{\theta r}\right)+\rho u_{r} F_{\phi r}-\rho u_{\phi} F_{r r} \\
\rho u_{\phi} F_{\theta r}-\rho u_{\theta} F_{\phi r}+\cot \theta\left(\rho u_{\theta} F_{\phi \theta}-\rho u_{\phi} F_{\theta \theta}\right)+\rho u_{r} F_{\phi \theta}-\rho u_{\phi} F_{r \theta} \\
\cot \theta\left(\rho u_{\theta} F_{\phi \phi}-\rho u_{\phi} F_{\theta \phi}\right)+\rho u_{r} F_{\phi \phi}-\rho u_{\phi} F_{r \phi} \\
0
\end{array}\right)
$$




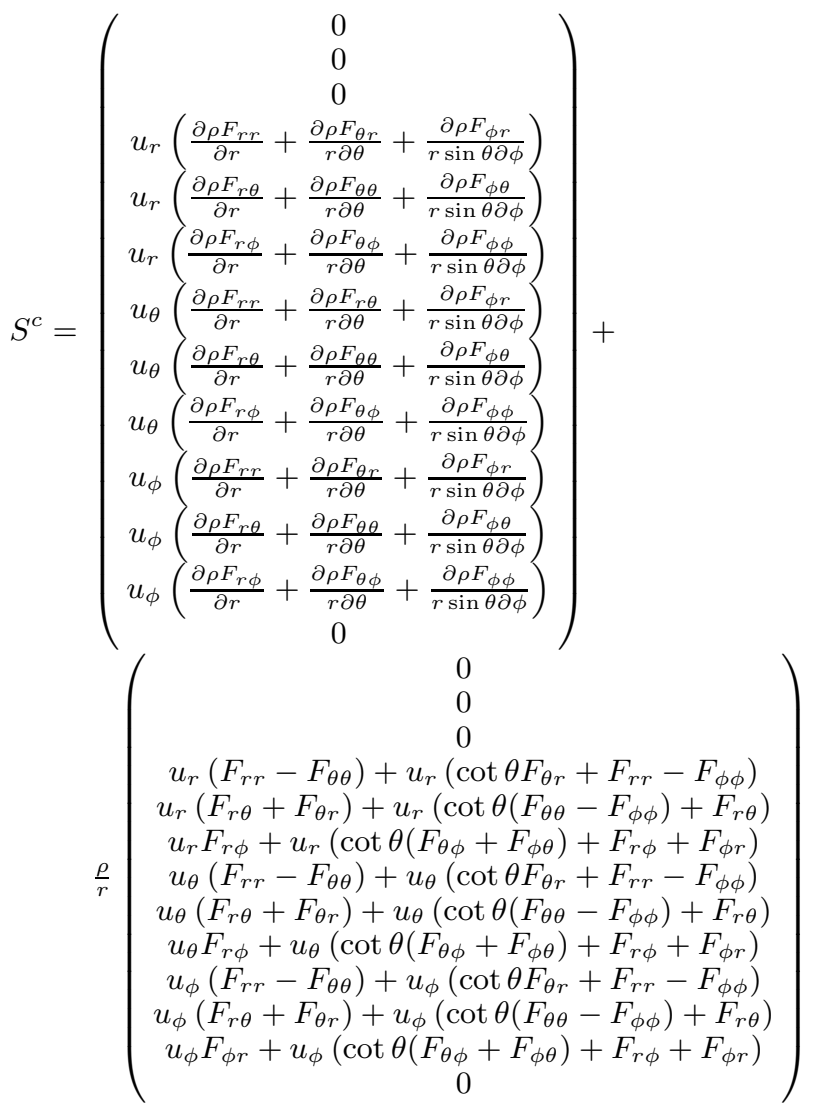

\section{REFERENCES}

1. Balsara DS, Shu C, Monotonicity preserving weighted essentially non-oscillatory schemes with increasingly high order accuracy, Journal of Computational Physics 2000; 160:405-452.

2. Barton PT, Drikakis D, Romenski E, Titarev VA, Exact and approximate solutions of Riemann problems in non-linear elasticity, Submitted to Journal of Computational Physics 2009.

3. Benson DJ, Computational methods in Lagrangian and Eulerian hydrocodes, Computer Methods in Applied Mechanics and Engineering 1992; 99:235-394.

4. Chen G.-Q, Levermore CD, Liu T.-P, Hyperbolic Conservation Laws with Stiff Relaxation Terms and Entropy, Communications on Pure and Applied Mathematics 1994; 47:787-830.

5. Eberle A, Characteristic Flux Averaging Approach to the Solution of Euler's Equations, in VKI Lecture Series, Computational Fluid Dynamics, 1987.

6. Godunov SK, Romenskii EI, Nonstationary Equations of Nonlinear Elasticity Theory in Eulerian Coordinates, Journal of Applied Mechanics and Technical Physics 1972; 13:868-884.

7. Godunov SK, Demchuk AF, Kozin NS, Mali VI Interpolation formulas for maxwell viscosity of certain metals as a function of shear-strain intensity and temperature, Journal of Applied Mechanics and Technical Physics 1974; 15:526-529.

8. Godunov SK, Romenski EI, Elements of Continuum Mechanics and Conservation Laws, Kluwer Academic/Plenum Publishers, 2003.

9. Jiang GS, Shu CW, Efficient implementation of weighted ENO schemes, Journal of Computational Physics 1996; 126:202-228.

10. Jin S, Runge-Kutta methods for hyperbolic conservation laws with stiff relaxation, it Journal of Computational Physics 1995; 122:51-67. 
11. Kluth G, Després B, Perfect plasticity and hyperelastic models for isotropic materials, Continuum Mechanics and Thermodynamics 2008; 20:173-192.

12. Kondaurov VI, Equations of elastoviscoplastic medium with finite deformations, Journal of Applied Mechanics and Technical Physics 1982; 23:584-591.

13. LeFloch PG, Olsson F, A second-order Godunov method for the conservation laws of nonlinear elastodynamics, Impact Comp. Sci. Eng 1990; 2:318-354.

14. Liu TG, Khoo BC, Yeo KS, Ghost fluid method for strong shock impacting on material interface, Journal of Computational Physics 2003; 190:651-681.

15. Merzhievskii LA, Samonin SA, Construction of the time dependence of the relaxation of tangential stresses on the state parameters of a medium, Journal of Applied Mechanics and Technical Physics 1980; 21:716724 .

16. Merzhievsky LA, Tyagel'sky AV, Relation of dislocation kinetics with dynamic characteristics in modelling mechanical behaviour of materials, Journal de Physique C3 1988; 49:457-466.

17. Miller GH, Colella P, A High-Order Eulerian Godunov Method for Elastic-Plastic Flow in Solids, Journal of Computational Physics 2001; 167:131-176.

18. Miller GH, Colella P, A Conservative Three-Dimensional Eulerian Method for Coupled Solid-Fluid Shock Capturing, Journal of Computational Physics 2002; 183:26-82.

19. Nourgaliev RR, Theofanous TG, High-fidelity interface tracking in compressible flows: Unlimited anchored adaptive level set, Journal of Computational Physics 2007; 224:836-866.

20. Pareschi L, Russo G, Implicit-Explicit Runge-Kutta Schemes and Applications to Hyperbolic Systems with Relaxation, Journal of Scientific Computing 2005; 25:129-155.

21. Plohr BJ, Sharp DH, A Conservative Formulation for Plasticity, Advances in Applied Mathematics 1992; 13:462-493.

22. Resnyanky AD, DYNA-modelling of the high-velocity impact problems with a split-element algorithm, International Journal of Impact Engineering 2002; 27:709-727.

23. Romenski EI, Thermodynamics and Hyperbolic Systems of Balance Laws in Continuum Mechanics, in Godunov Methods: Theory and Applications, edited by E. F. Toro. Kluwer Academic/Plenum Publishers, 2001

24. Shu C, Osher S, Efficient Implementation of Essentially Non-oscillatory Shock-Capturing Schemes, Journal of Computational Physics 1988; 77:439-471.

25. Titarev VA, Romenski E, Toro EF, MUSTA-type upwind fluxes for non-linear elasticity, International Journal of Numerical Methods in Engineering 2008; 73:897-926.

26. Vorobiev OY, Lomov IN, Shutov AV, Kondaurov VI, Ni AL, Fortov VE, Application of schemes on moving grids for numerical simulation of hypervelocity impact problems, International Journal of Impact Engineering 1995; 17:891-902.

27. Walter J, Yu D, Plohr BJ, Grove J, Glimm J, An algorithm for Eulerian front tracking for solid deformation, Stony Brook AMS Preprint 2000; SUNYSB-AMS-00-24.

28. Wang F, Glimm JG, Grove JW, Plohr BJ, A conservative Eulerian numerical scheme for elastoplasticity and application to plate impact problems, Impact of Computing in Science and Engineering 1993; 5:285-308.

29. Wilkins ML, Calculation of Elastic-Plastic Flow, UCRL Technical Report 1963; UCRL-7322. 